\title{
Unravelling overlaps and torsion-facilitated coupling using two-dimensional laser-induced fluorescence
}

David J. Kemp, Adrian M. Gardner, William D. Tuttle, and Timothy G. Wright*

School of Chemistry, University of Nottingham, University Park, Nottingham NG7 2RD, UK

*Tim.Wright@nottingham.ac.uk

\begin{abstract}
Two-dimensional laser-induced fluorescence (2D-LIF) spectroscopy is employed to identify contributions to fluorescence excitation spectra that arise from both overlapping bands and coupling between zero-order states (ZOSs). Evidence is found for the role of torsional motion in facilitating the coupling between vibrations that particularly involves the lowest-wavenumber out-of-plane vibrational modes. The experiments are carried out on jet-cooled $p$-fluorotoluene, where the molecules are initially in the lowest two torsional levels. Here we concentrate on the $390-420 \mathrm{~cm}^{-1}$ features in the $S_{1} \leftarrow S_{0}$ excitation spectrum, assigning the features seen in the 2D-LIF spectrum, aided by separate dispersed fluorescence spectra. The 2D-LIF spectra allow the overlapping contributions to be cleanly separated, including some that arise from vibrational-torsional coupling. Various coupling routes open up because of the different symmetries of the lowest two torsional modes; these combine with the vibrational symmetry to provide new symmetry-allowed vibration-torsion ('vibtor') interactions, and the role of the excited $m=1$ torsional level is found to be significant.
\end{abstract}




\section{Introduction}

Recently, there has been a concentrated effort at understanding the interactions between vibrations and torsions in substituted benzenes, which form vibration-torsional ('vibtor') levels. Work has focused on the monomethyl molecules, toluene and $p$-fluorotoluene $(p F T)$, and the dimethyl molecule, $p$-xylene ( $p \mathrm{Xyl})$. Toluene has been studied using both photoelectron spectroscopy (PES) and time-resolved PES (tr-PES), 1,2,3,4,5,6,7 zero-electron-kinetic-energy (ZEKE) spectroscopy, ${ }^{6,7,8}$ and twodimensional laser-induced fluorescence (2D-LIF). ${ }^{9,10,11,12,13}$ Work has been extended to the $p F T$ molecule where many of the interactions in the low-wavenumber region $\left(<430 \mathrm{~cm}^{-1}\right)$ of the $S_{1}$ state have been assigned employing both ZEKE ${ }^{14,15}$ and 2D-LIF spectroscopy. ${ }^{16}$ Earlier work includes: absorption studies by Cvitaš and Hollas ${ }^{17}$ and Seliskar et al., ${ }^{18}$ LIF and dispersed fluorescence (DF) spectroscopy by Okuyama et al., ${ }^{19} \mathrm{Ha}$ et al. ${ }^{20}$ and Parmenter, Zhao et al.; ${ }^{21,22,23,24}$ and resonanceenhanced multiphoton ionisation (REMPI) spectroscopy by Georgiev et al. ${ }^{25}$ and Hu et al. ${ }^{26}$, in both cases as part of a study of the pFT-Ar complex. Further work on higher-lying $\mathrm{S}_{1}$ levels has been undertaken using tr-PES, ${ }^{27}$ ZEKE ${ }^{28,29}$ and 2D-LIF spectroscopy. ${ }^{29}$ (A reassignment of the levels discussed in Ref. 27 has been presented in Ref. 29, and a reassignment of some of the levels discussed in Refs. 14 and 19 is presented in Refs. 15, 16 and 30.) The work of Gascooke, Lawrance and coworkers (GL) on toluene and $p \mathrm{FT}$ has provided the most-detailed description of the vibtor interactions occurring in both the $S_{0}$ and $S_{1}$ states and discussed the necessity of including such interactions in any detailed understanding of the spectroscopy of these molecules. We tackled the $p$-xylene ( $p$ Xyl) molecule recently ${ }^{31,32}$ and showed that the vibtor interactions, although now involving a two-rotor system, were very similar to those of toluene and $p \mathrm{FT}$. Overall, these vibtor interactions provide increased pathways for energy dissipation between the molecular motions and so can lead to the possibility of rapid intramolecular vibrational redistribution (IVR) at even quite low wavenumbers, and this has been compared and contrasted in recent papers focused on toluene, $p$-difluorobenzene ( $p \mathrm{DFB})$ and $p \mathrm{FT},{ }^{6}$ as well as $p$ DFB, $p$-chlorofluorobenzene ( $p$ ClFB), $p \mathrm{FT}$ and $p$ Xyl. ${ }^{33}$

Recently, ${ }^{29}$ we showed that the vibtor levels at $\sim 845 \mathrm{~cm}^{-1}$ associated with the methyl torsion and the first overtone of an out-of-plane vibration in $p F T$ were opening up new routes to interactions involving vibrations of different symmetry. This involved interactions between an excited torsional level of an overtone with vibtor levels formed by combinations with a set of levels at $\sim 400 \mathrm{~cm}^{-1}$. In the present work, we revisit those levels close to $\sim 400 \mathrm{~cm}^{-1}$ in the $S_{1}$ state of $p$ FT and examine the interactions using 2D-LIF spectroscopy. The present work is an extension to recently-reported work by GL, ${ }^{16}$ which 
focused on a detailed understanding of the $S_{1}$ region $<400 \mathrm{~cm}^{-1}$, highlighting interactions occurring for vibtor levels involving the three lowest-wavenumber vibrations.

\section{Experimental}

The 2D-LIF apparatus has been described recently. ${ }^{29}$ The vapour above room temperature parafluorotoluene (99\% purity, Alfa Aesar) was seeded in $\sim 5$ bar of Ar and the gaseous mixture passed through a General Valve pulsed nozzle $(750 \mu \mathrm{m}, 10 \mathrm{~Hz}$, opening time of 180-210 $\mu \mathrm{s})$ to create a free jet expansion. This was intersected at $X / D \sim 20$ by the frequency-doubled output of a single dye laser, operating with C540A. The fluorescence was collected, collimated and focused onto the entrance slits of a 1.5 m Czerny Turner spectrometer (Sciencetech 9150) operating in single-pass mode, dispersed by a 3600 groove/mm grating, and then $\sim 300 \mathrm{~cm}^{-1}$ windows of the dispersed fluorescence were collected by a CCD camera (Andor iStar DH334T). At a fixed grating angle of the spectrometer, the wavenumber of the excitation laser was scanned, and at each excitation wavenumber the image was accumulated for 2000 laser shots. This produced a 3D surface of fluorescence intensity versus the excitation laser wavenumber and the wavenumber of the emitted and dispersed fluorescence, termed a 2D-LIF spectrum. ${ }^{13}$

We have also recorded separate DF spectra with higher averaging to get better signal to noise than simply taking a vertical slice through the 2D-LIF image. These DF spectra were recorded with the same spectrometer as for the 2D-LIF spectra, and were recorded three times accumulating over 5000 shots each time, and an average taken.

\section{Results and discussion}

\subsection{Nomenclature and labelling}

\subsubsection{Vibrational labelling}

In previous work, authors have used a combination of Wilson ${ }^{34} /$ Varsány $^{35}$ and Mulliken ${ }^{36} /$ Herzberg $^{37}$ notation. As we have previously noted, Wilson notation is inappropriate because of the large change in the forms of the vibrations, from benzene to $p \mathrm{FT}^{38}$ also, the Varsányi notation is inconsistent in its treatment of different molecules (and rather confusingly also uses Wilson-type labels). We have also 
pointed out ${ }^{8,15}$ that previous usage of Mulliken labels is inconsistent as the list includes the $\mathrm{CH}_{3}$ localised vibrations, yet the $C_{2 v}$ point group is used for the numbering. We shall comment on previous assignments alongside ours in terms of the $D_{i}$ labels from Ref. 38 , which are also the labels used by ourselves and $\mathrm{GL}$ in published work on toluene and $p \mathrm{FT}$. (The various labels used in previous work have been included in Table 1 to aid the reader in referring between the different studies.)

Some assignments will involve vibtor levels and for these, the $G_{12}$ molecular symmetry group (MSG) is appropriate, and so we shall use this throughout the present work. In addition, torsional levels will be labelled via their $m$ quantum number, noting that the $m$ number is signed, but usually only the positive value is employed. The reader may find it useful to refer to previous work ${ }^{10,11,12,15,31}$ if they are not familiar with these labels. The correspondence between the $C_{2 v}$ point group labels and the $G_{12}$ MSG ones are given in Table 2. To calculate the overall symmetry of a vibtor level, it is necessary to use the corresponding $G_{12}$ label for the vibration (Table 2), and then find the direct product with the symmetry of the torsion, noting that a $D_{3 h}$ point group direct product table can be used, since the $G_{12}$ MSG and the $D_{3 h}$ point group are isomorphic. The symmetries of the $m$ levels are also given in Table 2 .

Under the free-jet expansion conditions employed here, the molecules are all expected to be cooled to their zero-point vibrational level; note, however, that owing to nuclear-spin and rotational symmetry, the molecules can be in one of the $m=0$ or $m=1$ torsional levels. ${ }^{31,39}$

\subsubsection{Vibrational coupling}

Each molecule has a set of vibrational normal modes that, within the harmonic approximation, constitute the various allowed coherent motions of the atoms that keep the centre of mass stationary with respect to a system of axes that rotates and translates with the molecule. In principle, the vibrations are non-interacting no matter how close the vibrations are in wavenumber, as long as we remain within the harmonic model. When comparing between similar molecules, then these normal modes might be expected to look very similar; however, it is found that the effect of changing the mass of one of the substituents can have quite a dramatic effect on the form and wavenumber of some of the vibrations. As a consequence, even within the harmonic model, the normal modes of one molecule may be quite different from those of a related one, such as between benzene and fluorobenzene, ${ }^{40,41}$ and between benzene and $p D F B .{ }^{38}$ There will then be various combination and overtone levels associated with these. 
If we now include anharmonicity then, if all of the vibrational levels of a particular molecule are far enough apart in wavenumber, the interactions between them will be minimal. In this case, the eigenstates will correspond to these vibrations, each of which will be an anharmonic vibration corresponding to a particular normal mode. The difference between the well-separated anharmonic normal modes and the harmonic normal modes can be termed 'diagonal anharmonicity'. If, however, an anharmonic fundamental is close in wavenumber to one or more levels that has the same overall symmetry, then 'off-diagonal' anharmonic interactions can occur. The non-interacting levels are termed zero-order states (ZOSs), and coupling between these leads to the formation of eigenstates that are linear combinations of these, and will be at different wavenumbers to the original ZOSs. ${ }^{37}$ This is most likely to occur when the ZOSs are energetically close together. For two interacting states, this is termed a Fermi resonance, ${ }^{42}$ while for more than two levels we term this a complex Fermi resonance. For molecules that contain a hindered internal rotor, the ZOSs can also be torsional or vibtor levels and these can become involved in the eigenstates, thus involving further degrees of freedom. In general, these interactions lead to eigenstates that involve more widespread atomic motions and so the ZOS mixing promotes dispersal of energy through the molecule.

In electronic spectroscopy, if we assume a non-coupled picture initially, then a vibrational or vibtor ZOS can be bright (i.e. it has a significant transition intensity) or dark (i.e. it has no, or a very small transition intensity); these are often termed zero-order bright (ZOB) states and zero-order dark (ZOD) states, respectively. Following interaction, a particular eigenstate may be composed of a mixture of ZOB and ZOD state character. In the simplest case of one ZOB state interacting with one ZOD state, then two eigenstates will be formed (corresponding to a sum and difference of the ZOB and ZOD states - the classic Fermi resonance ${ }^{35,42}$ ), both of which will appear in the electronic spectrum, by virtue of the ZOB state character. Hence, as well as shifts in the observed positions of spectral lines from those expected, the interaction can give rise to the appearance of 'extra' spectral lines. In the experiments performed here, nanosecond lasers are employed, and individual eigenstates (ignoring rotational energy levels) can usually be picked out in the spectrum. By observing projections of these eigenstates onto those in another state either by DF, or by photoionisation and recording photoelectron spectra, insight into the ZOS make-up of the excited state eigenfunctions can be determined.

Such couplings are only expected to be significant for small changes, $\Delta v \leq 3$, of the vibrational quantum number, and also for changes, $\Delta m$, of $0, \pm 3$ or \pm 6 in the torsional quantum number in descending order of likely strength. ${ }^{11,15,31,43,44}$ 


\subsubsection{Transitions}

When designating excitations, we shall generally omit the lower level, since it will be obvious from the jet-cooled conditions; similarly, for emissions, we shall omit the upper level, as that will be obvious from the excitation and context. In the usual way, vibrational transitions will be indicated by the ordinal number, $i$, of the $D_{i}$ vibration, followed by the number of quanta; torsional transitions will be indicated by $m$ followed by its value. Finally, vibtor transitions will be indicated by a combination of the vibrational and torsional transition labels. If no $m$ values are specified, then transitions involving both $m=0$ and $m=1$ levels occur, whose transition wavenumbers are expected to be coincident at the present resolution. The wavenumbers of the levels will be given with respect to the relevant zeropoint level in each state, but noting that some excitations will originate from the $m=1$ level in $S_{0}$ and those transition energies are given with respect to that level, as usual. As has become common usage, we will generally refer to a level using the notation of a transition, with the level indicated by the specified quantum numbers. Also, since in the present work all of the interactions between ZOSs appear to be weak, the eigenstates are usually referred to as the overwhelmingly dominant contribution from one of the ZOSs, with the context implying if a small admixture of other ZOSs is present.

We give two examples: (i) $\left(14^{2} m^{0}, 14_{1} 19_{1} m_{3(+)}\right)$ represents an excitation from the $\mathrm{S}_{0}$ zero-point $m=0$ level to the $m=02 D_{14}$ level in the $S_{1}$ state, followed by emission from this $S_{1}$ level to the $m=3(+)$ level of the $D_{14}+D_{19}$ combination level in $S_{0}$; and (ii) $\left(14^{2}, 9_{1}\right)$ represents a dual excitation from the $m=0$ and $m=1$ levels of the $S_{0}$ zero-point level, followed by dual emission to the corresponding $m$ levels of the $D_{9}$ level in the $S_{0}$ state - note that these would be coincident with our resolution.

\subsection{Overall comments on the $S_{1} \leftarrow S_{0}$ spectrum}

We have discussed the $0-830 \mathrm{~cm}^{-1}$ region of the $\mathrm{S}_{1} \leftarrow \mathrm{S}_{0}$ excitation spectrum of $p$ FT employing REMPI and ZEKE spectroscopy in our previous work. ${ }^{15,28}$ In addition we have discussed a feature at $~ 845 \mathrm{~cm}^{-1}$ using REMPI, ZEKE and 2D-LIF spectroscopy. ${ }^{29}$ In Figures 1(a) and 1(b) we show expanded views of the region of the $390-415 \mathrm{~cm}^{-1}$ region $\mathrm{S}_{1} \leftarrow \mathrm{S}_{0}$ spectrum, which is the centre of attention here, recorded using both REMPI and 2D-LIF spectroscopy (integrated in the latter case - see below); it can be seen that they are very similar. 


\subsection{Activity expected in the $2 D$-LIF and DF spectra}

For eigenstates that are dominated by a single ZOS, we expect to see a dominant $\Delta(v, m)=0$ band as the strongest contributor to the fluorescence from such a level. Associated with this will be other Franck-Condon (FC)-active vibrational transitions or symmetry-allowed vibtor contributions arising from changes in geometry and/or vibrational frequencies and/or torsional potentials - for convenience, we refer to both of these as 'FC transitions' or 'FC activity' in the below. Occasionally, if there are large changes between the potentials, then it is possible that the $\Delta(v, m)=0$ band will not be the most intense. It is possible to see weaker transitions associated with $\Delta m= \pm 3$ and, even weaker, transitions associated with $\Delta m= \pm 6$.

If an eigenstate is made up of more than one ZOS, then we expect contributions from each ZOS to appear in the spectrum. However, this assumes that there is a significant difference between the ZOS make-up of the eigenstates in both electronic states. Indeed, if the eigenstate make-up were identical in these two states, than we would just see a spectrum dominated by the $\Delta(v, m)=0$ band corresponding to transitions between the particular eigenstate in both electronic states. Usually, however, there is a significant difference in the make-up and then for a simple Fermi resonance we would expect to see both $\Delta(v, m)=0$ bands, with that of one ZOS dominating from one FR component, and the reverse for the second component.

In the 2D-LIF spectrum recorded across the $390-415 \mathrm{~cm}^{-1}$ range of excitation wavenumber (see Figure 2), we can see that there are two vertical strips of activity localised across different ranges of the excitation wavenumber. Previous ZEKE work $^{14}$ has shown that the higher-wavenumber excitation feature at $408 \mathrm{~cm}^{-1}$ in the REMPI/LIF spectrum in Figure 1 is largely associated with exciting $11^{1}$, while the lower-wavenumber feature corresponds to an overlapped $14^{2} / 29^{1}$ pair of transitions, with the $14^{2}$ transition to slightly lower wavenumber. Closer inspection of the 2D-LIF spectrum in Figure 2 (and see later) indicates that indeed the lower-wavenumber vertical strip of activity is largely split into two subsets, separated in excitation wavenumber. Those slightly higher in wavenumber are consistent with being associated mainly with $29^{1}$ and those slightly lower with $14^{2}$. This activity will be discussed as part of the assignments below. It will also be noticed that the various bands have different profiles made up from the underlying rotational structure. Bands that are associated with $a_{1}{ }^{\prime \prime}$ levels (such as the $m=0$ levels of the $D_{29}$ vibration) are of $a$-type, while those of $a_{1}{ }^{\prime}$ levels (such as the $m=0$ levels of the $D_{11}$ vibration) are of $b$-type. ${ }^{13,16}$ The band type may not always be obvious in cases of overlapping bands, or when rotationally-dependent interactions occur. 
To identify the position of bands in the 2D-LIF spectrum in Figure 2, we refer to the pair of respective excitation and emission relative wavenumbers, with an assignment being given in terms of the pairs of respective transitions, as has been used by GL. ${ }^{13,16}$ From these considerations, it is straightforward to identify the most intense bands in the spectrum, and these are in agreement with previous assignments. ${ }^{14,15,16}$ The band at $(399,424) \mathrm{cm}^{-1}$ is predominantly from the $\left(29^{1}, 29_{1}\right) \Delta v=0$ transition; the band at $(409,453) \mathrm{cm}^{-1}$ is associated predominantly with $\left(11^{1}, 11_{1}\right)$; and the band at $(397,824)$ $\mathrm{cm}^{-1}$ predominantly with $\left(14^{2}, 14_{2}\right)$. Each of these bands contains separate transitions between the respective $m=0$ level and the $m=1$ level associated with each vibrational state; these cannot be discerned at the present resolution. In Figures 3-5 we present DF spectra at positions that correspond to excitation of $0^{0}$ and those corresponding to the eigenstates dominated by $14^{2}, 29^{1}$ and $11^{1}$ - noting that the $14^{2}$ and $29^{1}$ transitions have overlapped rotational profiles and so their separation is not wholly complete. These DF spectra are the equivalent of taking a vertical cut-through of the 2D-LIF image; however, these traces were recorded separately by averaging over a greater number of laser shots. The assignments of most of the relatively intense bands are straightforward and these are given on the figures and will be summarised briefly below. Some other more 'interesting' assignments will be discussed separately.

\subsection{Assignments}

\subsubsection{Initial discussion}

We will now give an overview of the assignments of the main transitions initially by reference to the DF spectra in Figures $3-5$, obtained by exciting at the origin, $0^{\circ}$, and positions corresponding to levels expected ${ }^{14,15,16}$ to be dominated by $14^{2}, 29^{1}$ and $11^{1}$; we show the $0-1390 \mathrm{~cm}^{-1}$ region of the DF spectra across these figures. Later, we shall discuss some particular features of the DF and 2D-LIF spectra further, highlighting the information that can be deduced regarding any interactions between the ZOSs.

When exciting via $0^{0}$, in the region between $0-390 \mathrm{~cm}^{-1}$ (see Figure 3 ) there is a wealth of torsional and vibtor activity in the DF spectrum that has been discussed in depth by Gascooke et al., ${ }^{16}$ and the reader is referred to that work. Unfortunately, the signal-to-noise is not sufficient to see any of these levels when exciting via $14^{2}, 29^{1}$ or $11^{1}$. 
In the regions of the DF spectra in Figures 4 and 5, we also see bands associated with totallysymmetric, $a_{1}{ }^{\prime}$ fundamentals, whose wavenumbers largely agree with those discussed and presented in Ref. 38. (Revised values for $D_{20}, D_{19}$ and $D_{30}$ have been presented in the 2D-LIF study of Gascooke et al. ${ }^{16}$ and are in better agreement with the calculated values ${ }^{38}$ than the previous ones - see Table 1.) We also see several bands that arise from Herzberg-Teller (HT) activity, and involve in-plane vibrations of $a_{1}$ " symmetry. In the region scanned, we have assigned fundamental bands associated with $29_{1}$, $11_{1}, 28_{1}, 10_{1}, 9_{1}, 7_{1}, 6_{1}$ and $5_{1}$. We also see a number of first overtone bands associated with out-ofplane modes: $20_{2}, 19_{2}, 18_{2}$ and $14_{2}$. There are also a wealth of combination bands involving pairs of out-of-plane $a_{2}{ }^{\prime}$ and $a_{2}{ }^{\prime \prime}$ vibrations (and so totally-symmetric, $a_{1}{ }^{\prime}$, overall). The symmetry-allowed $28_{1}$ emission is seen following $29^{1}$ absorption, but a band at the same wavenumber also seen when exciting $0^{\circ}$. Although this could arise from similar HT activity that leads to the observation of the $29_{1}$ emission, another possible assignment is $18_{12} 20_{1}$.

From the above, a large number of fundamental vibrational wavenumbers in the $\mathrm{S}_{0}$ state can be derived, and these are included in Table 1 alongside the previous experimental and calculated values. As well as these, there are various other expected combinations: for example, with $11_{1}$ and $9_{1}$. Clearly, to higher wavenumber more and more possibilities exist for an assignment; however, mostly a satisfactory one can be reached from earlier activity and the previous experimental and calculated values. ${ }^{38}$ As a general point, the assignment of most bands is confirmed by their changing intensities when exciting from the different $S_{1}$ levels, so that combinations involving $11_{1}$ are particularly strong when emitting from $11^{1}$, for example. We also note that totally-symmetric fundamentals are particularly prominent when exciting via $0^{0}$.

\subsubsection{Main activity the $2 D$-LIF spectrum}

We initially comment that from the section of the DF spectrum in Figure 4. We can see that when exciting to $0^{0}$, the $11_{1}$ band is intense, while the $29_{1}$ band is significantly weaker, with the $14_{2}$ band being somewhere in between. It is clear from the significant separation of the bands, that there is unlikely to be any interaction between the $29_{1}, 11_{1}$ and $14_{2}$ levels in $S_{0}$. The spectrum from $11^{1}$ shows a strong $11_{1}$ band, confirming its assignment; also, there is a very weak $14_{2}$ band and no clearly discernible $29_{1}$ band. The separate band that appears at $438 \mathrm{~cm}^{-1}$ is assigned as arising from $29_{1} m_{2}$, which is a symmetry-allowed emission from $11^{1} \mathrm{~m}^{1}$ (see later). 
From $29^{1}$ we see a strong $29_{1}$ band in the DF spectrum in Figure 4, and although there are apparent weak $11_{1}$ and $14_{2}$ bands, these appear to be arising from a simultaneous excitation of $14^{2}$ via the overlap of the rotational envelopes of the $29^{1}$ and $14^{2}$ bands. Lastly, from $14^{2}$, we see a very strong $14_{2}$ band, and a very weak $11_{1}$ band; the significant $29_{1}$ band appears to arise from the $14^{2} / 29^{1}$ rotational envelope overlap. It is unfortunate that the partial overlap between the $29^{1}$ and $14^{2}$ bands obfuscates clear conclusions regarding possible interactions between the $14^{2}, 29^{1}$ and $11^{1}$ levels further insight arises from the 2D-LIF spectra, which are now discussed in more detail.

An overview of the main 2D-LIF activity can be seen in Figure 2, but we now concentrate on expanded views of the main $\Delta(v, m)=0$ regions, with the first focus of attention on the region shown in Figure 6. The comments above regarding the assigned activity in the DF spectra clearly also hold, but it is also apparent that there is more information in the 2D-LIF image. As presented, activity from emission to the same $S_{0}$ level appears horizontally across the 2D-LIF images, while emission to various $S_{0}$ levels from exciting a particular $S_{1}$ level appears vertically at that excitation number.

Emission activity from $11^{1}$ is evident on the right-hand side of Figures 2 and 6 , concentrated across the excitation numbers $405-410 \mathrm{~cm}^{-1}$ and dominated by the $\left(11^{1}, 11_{1}\right)$ band. On the left-hand side of Figure 2, we clearly have two main subregions of emission activity, associated with exciting the level that is predominantly $29^{1}$ and, to slightly lower wavenumber, emission from the one that is predominantly $14^{2}$; these subregions are each dominated by their respective $\Delta(v, m)=0$ bands. In deducing the assignments of other bands arising from emission from these upper states, we take account of the fact that such bands are expected to have a similar width of activity as the $\Delta(v, m)=0$ band across the region. As well as $\left(11^{1}, 29_{1} m_{2}\right)$ activity, we also see a weak but distinct $\left(29^{1} m^{2}, 29_{1} m_{2}\right)$ band at $(412,438) \mathrm{cm}^{-1}$ in the 2D-LIF spectrum in Figure 6 together with broad activity across this region.

Immediately apparent from Figure 6 is that although there is a strong $\left(29^{1}, 29_{1}\right)$ band, there is no clear evidence for a $\left(11^{1}, 29_{1}\right)$ band, suggesting no significant interaction between $29^{1}$ and $11^{1}$, as mentioned above when discussing the DF spectra. In Figure 7 we show traces where we have integrated vertical strips of activity across the 2D-LIF spectrum for the main bands. It can be seen that there is very weak $29_{1}$ activity in the region of $11^{1}$, and a correspondingly small amount of $11_{1}$ activity when exciting via $29^{1}$. However, examining the activity at various emission wavenumbers shows that these observations are artefacts of the integrations, which are picking up some of the weak, broad activity from the main $\Delta(v, m)=0$ bands. As a consequence, we conclude that there is no evidence for interaction between 
$29^{1}$ and $11^{1}$. Also apparent in the 2D-LIF spectrum in Figure 6 is a weak band at $(399,457) \mathrm{cm}^{-1}$; since this seems to be associated with $29^{1}$, we assign this to the symmetry-allowed $\left(29^{1} m^{0}, 14_{1} m_{3(-)}\right)$ transition.

In Figure 8(a), we see the strong $\left(14^{2}, 14_{2}\right)$ band, which is clearly displaced from that of the $\left(29^{1}, 29_{1}\right)$ band, and a weak $\left(14^{2}, 11_{1}\right)$ band. The latter is consistent with the $\left(11^{1}, 14_{2}\right)$ band seen in this figure. These could arise from FC activity, or be evidence that the $11^{1}$ and $14^{2}$ levels are weakly coupled in the $S_{1}$ state; the integrated traces in Figure 7 are also consistent with these. Although the shoulder on the $29_{1}$ trace is consistent with suggesting some $29_{1}$ emission from $14^{2}$, this is thought to arise from underlying rotational structure, since there is no corresponding shoulder on the low-wavenumber side of the $14_{2}$ band, suggesting no $29_{1}$ emission from $14^{2}$. This is also evident in the 2D-LIF image in Figure 6 , where the $\left(29^{1}, 29_{1}\right)$ band is seen to be localised, and there is no extension of this band to lower wavenumber, as would be expected if there were a $\left(14^{2}, 29_{1}\right)$ contribution. Overall, we conclude that there is no significant interaction between the $14^{2}$ and $29^{1}$ levels.

We will discuss the $489 \mathrm{~cm}^{-1}$ band - see Figure 6 - in Section 3.4.3.2.

As well as the three aforementioned ZOSs, in our ZEKE study ${ }^{15}$ we deduced the involvement of another: $14^{1} \mathrm{~m}^{6(-)}$ and this was confirmed by Gascooke et al. ${ }^{16}$ in their recent 2D-LIF study. In those studies, it was inferred that the $14^{2} \mathrm{~m}^{0}$ and $14^{1} \mathrm{~m}^{6(-)}$ states were coupled. In our ZEKE study, ${ }^{15}$ we concluded that we saw the second component of the interaction in the $S_{1}$ state at $364 \mathrm{~cm}^{-1}$, but we subsequently noted ${ }^{29}$ that unpublished 2D-LIF spectra, now reported herein, did not support this, and this is in agreement with the deductions of Gascooke et al. ${ }^{16}$ that the interaction is very localised. We show an expanded view of this region of the 2D-LIF spectrum in Figure 9, where the $\left(14^{1} \mathrm{~m}^{6(-)}, 14_{1} \mathrm{~m}_{6(-)}\right)$ band may be clearly seen to have a rather unusual band profile, in agreement with that presented in Ref. 16, which is better resolved therein.

In Figure 10, we show an expanded view of the 2D-LIF spectrum in a region to lower wavenumber than that of Figure 2, but covering an excitation wavenumber of $0^{0}+364 \mathrm{~cm}^{-1}$. It can be seen that there is no evidence of emission to the $618 \mathrm{~cm}^{-1}$ level in this region. The deduction from the ZEKE spectrum ${ }^{15}$ arose from a ZEKE band at $538 \mathrm{~cm}^{-1}$ seen when exciting at $364 \mathrm{~cm}^{-1}$. From Figure 10, we see that there is a $2 \mathrm{D}$-LIF band at $(365,635) \mathrm{cm}^{-1}$, which can be assigned to $\left(14^{1} 20^{1} \mathrm{~m}^{4}, 14_{1} 20_{1} \mathrm{~m}_{4}\right)$, with the wavenumber of the upper state being consistent with the energy-level diagram presented in Ref. 16, and the lower level also in a reasonable position. In the cation, this level would be expected at $\sim 540$ 
$\mathrm{cm}^{-1}$ and so is consistent with the observed ZEKE band. Two other ZEKE bands were seen when exciting at this wavenumber. One was assigned to $30^{1} \mathrm{~m}^{3(+)}$, consistent with the observed 2D-LIF band seen in Ref. 16, and a second was assigned to $19^{1} 20^{1} \mathrm{~m}^{2}$. In the 2D-LIF spectrum the band at $\sim(366,501) \mathrm{cm}^{-1}$ pleasingly fits the assignment as the $\Delta(v, m)=0$ band $\left(19^{1} 20^{1} m^{2}, 19_{1} 20_{1} m_{2}\right)$.

The $14^{1} \mathrm{~m}^{6(-)}$ band would not be expected to be very bright exciting from a $m=0$ 'pure' vibrational level, since $\Delta m=6$; and indeed this band is extremely weak in the DF spectra from $0^{\circ}$ (Figure 4), and not seen via $11^{1}$. (Although it appears that there is activity to this level from $29^{1}$, this arises from the overlapping rotational profile with $14^{2}$.) The $14^{1} \mathrm{~m}^{6(-)}$ band is seen strongly from $14^{2}$ and its intensity profile across the spectrum is very similar to that of the $14^{2}$ band - see Figure 7, supporting the suggestion that these are intricately coupled. The overall coupling seems to be weak, however, as the two FR components are overlapped and so have not moved apart in energy; $\mathrm{GL}^{16,45}$ have concluded that the coupling between these levels depends on the rotational levels of the two ZOSs moving in and out of resonance.

It is interesting to observe that in the $S_{1}$ state ${ }^{16}$ the $m^{6(-)}$ and $14^{1} m^{1}$ levels are close together, at 197.8 $\mathrm{cm}^{-1}$ and $201.4 \mathrm{~cm}^{-1}$. Of course, $\mathrm{m}^{6(-)}$ can only interact with $14^{1} \mathrm{~m}^{0}$ but the corresponding band was noted as being too weak to see; ${ }^{16}$ however, the difference in transition wavenumber is expected to be similar at $\sim 3.6 \mathrm{~cm}^{-1}$. The fact that the $14^{2}$ and $14^{1} \mathrm{~m}^{6(-)}$ states are close to coincident in $\mathrm{S}_{1}$ suggests that the interactions between ZOSs in the two different wavenumber regions are different.

\subsubsection{Specific features}

3.4.3.1 Additional features around the $\left(14^{2}, 14_{2}\right)$ band. When we look at the $\left(14^{2}, 14_{2}\right) \Delta(v, m)=0$ band in more detail - see Figure $8(b)$ - we can see indications of two other contributions that are located close to the $29^{1}$ excitation energy: $(399,818) \mathrm{cm}^{-1}$ (labelled A) and $(399,829) \mathrm{cm}^{-1}$ (labelled B). The $14_{2} m_{0}$ and $14_{2} m_{1}$ origins are located at $822.8 \mathrm{~cm}^{-1}$ and $823.5 \mathrm{~cm}^{-1}$ according to $\mathrm{GL}^{16}$ but the two bands are not separable with our resolution. Assuming that the two extra bands indeed arise from exciting $29^{1}$, suggested assignments are $\left(29^{1} \mathrm{~m}^{1}, 29_{1} 30_{1} m_{4}\right)$ and $\left(29^{1} \mathrm{~m}^{1}, 14_{1} 29_{1} m_{1}\right)$. These extra features are evident in Figure S4 of Ref. 16 , but the higher wavenumber emission band (A) was associated with $o$ - and $m$ - isomers of the ${ }^{13} \mathrm{C} p \mathrm{FT}$ isotopologue, with the lower one (B) not being assigned, the profile of band A suggests the isotopomer assignment is the more reliable. ${ }^{45}$ 
Another possible source for the emission at $829 \mathrm{~cm}^{-1}$ (band B) would be from the $14_{2} m_{1} \ldots 14_{1} 19_{1} m_{4}$ interaction in the $\mathrm{S}_{0}$ state, analogous to the $14_{1} m_{1} \ldots 19_{1} m_{4}$ interaction discussed by GL. ${ }^{16}$

3.4.3.2 $489 \mathrm{~cm}^{-1}$ emission. We now consider the band at an emission wavenumber of $489 \mathrm{~cm}^{-1}-$ see Figure 6. The profile of this band suggests that it could be made up of two contributions: the more intense seems to be located close to the excitation profile of the $\left(29^{1}, 29_{1}\right)$ band, while the weaker part extends across the $\left(14^{2}, 14_{2}\right)$ region; there is only the faintest trace of activity at the $11^{1}$ excitation position. Being so low in wavenumber, there are not too many viable $\Delta(v, m)=0$ assignments that match both the excitation and emission wavenumbers. To this end we can rule out the following possibilities on the basis of the expected position of the $S_{1}$ level, even though the corresponding $S_{0}$ level would be at about the correct wavenumber: $20^{3} \mathrm{~m}^{3(-)}, 20^{1} \mathrm{~m}^{8}$, and $18^{1} \mathrm{~m}^{1}$. We find we have two main contenders for a $\Delta(v, m)=0$ band: $\left(19^{1} m^{5}, 19_{1} m_{5}\right)$ and $\left(20^{2} m^{6(+)}, 20_{2} m_{6(+)}\right)$, which we now discuss.

The $19^{1} \mathrm{~m}^{5}$ level would have the same symmetry as $29^{1} \mathrm{~m}^{1}$ and so would potentially be a candidate via an interaction with that level. However, it is somewhat too low in both $S_{1}$ (expected at $\sim 383 \mathrm{~cm}^{-1}$ ) and So (expected at $\sim 464 \mathrm{~cm}^{-1}$ ). ${ }^{16}$ Hence, even though it fits (expected $\sim 403 \mathrm{~cm}^{-1}$ ) the ZEKE band observed at $400 \mathrm{~cm}^{-1}$ (Ref. 15) when exciting close to $29^{1}$, we reject this assignment as it is expected too far away from the actual absorption and emission positions.

We would expect the $20_{2} m_{6(+)}$ band to appear at $492 \mathrm{~cm}^{-1}$, which is in very good agreement with the observed band, and although we might expect the excitation to be at $417 \mathrm{~cm}^{-1}, \mathrm{GL}^{16}$ suggest this should be at $\sim 398 \mathrm{~cm}^{-1}$ following vibtor coupling, which would be in line with the band's position. The associated ZEKE band would be expected at $\sim\left(222+189=410 \mathrm{~cm}^{-1}\right)$, and indeed we suggested this as an assignment of the observed broadish band at $\sim 400 \mathrm{~cm}^{-1}$ in Ref. 15 , and so $20_{2} m_{6(+)}$ activity is a viable contributor to the $489 \mathrm{~cm}^{-1}$ band. The $20^{2} \mathrm{~m}^{6(+)}$ level has the correct symmetry to interact with the $14^{2} m^{0}$ level, which would involve a $\Delta v=4, \Delta m=6$ interaction. Hence, there is a mechanism by which the $20^{2} \mathrm{~m}^{6(+)}$ band can gain intensity from $14^{2} \mathrm{~m}^{0}$ that is consistent with the presence of the weak part of this band at the $14^{2}$ excitation position and the ZEKE spectrum of Ref. 15 . We can then assign the brighter part of the band to the $\Delta(v, m)=0$ transition. We thus suggest an assignment of the $489 \mathrm{~cm}^{-1}$ emission band to $20_{2} m_{6(+)}$, with intensity arising from the $14^{2} m^{0} \ldots 20^{2} m^{6(+)}$ interaction. 
3.4.3.3. $500-750 \mathrm{~cm}^{-1}$ bands. In Figure 9, we also see weak emissions in the range $500-600 \mathrm{~cm}^{-1}$ when exciting across $395-405 \mathrm{~cm}^{-1}$. A very weak band at $(408,502) \mathrm{cm}^{-1}$ (which is not clearly discernible in the presented figures, but can be seen in expanded versions) is likely $\left(11^{1}, 11_{1} m_{3(+)}\right)$, while the extremely weak (unmarked) band at $(399,522) \mathrm{cm}^{-1}$ is plausibly $\left(29^{1}, 19_{1} 20_{1} m_{3(+)}\right)$. The DF spectrum in Figure 4 provides confirmation that there are two weak bands at $(398,538) \mathrm{cm}^{-1}$ and $(399,540) \mathrm{cm}^{-1}$ - see Figure 9. They have possible assignments of $\left(14^{2} m^{1}, 14_{1} m_{5}\right)$ and $\left(29^{1} m^{1}, 11_{1} m_{4}\right)$, respectively. We also see that there is a very weak band (not shown) in the DF spectrum at $546 \mathrm{~cm}^{-1}$, seen when exciting via $11^{1}$ and $0^{0}$, and a likely assignment of this is to $18_{1} m_{3(-)}$.

The band at $(399,558) \mathrm{cm}^{-1}$ in Figure 9 is assigned as $\left(29^{1}, 14_{1} 20_{1}\right)$ and is consistent with the observation of the $\left(0^{0}, 14_{1} 20_{1}\right)$ band assigned by Gascooke et al. Although the band at $(398,576) \mathrm{cm}^{-1}$ could be assigned as $\left(14^{2}, 20_{4}\right)$ or $\left(29^{1} \mathrm{~m}^{1}, 20_{1} 29_{1} m_{1}\right)$, it seems to be located close to centre of the $14_{1} m_{6(-)}$ band; additionally, the DF spectrum suggests $14^{1}$ involvement, and so an assignment to $\left(14^{2} \mathrm{~m}^{1}, 14_{1} 20_{1} m_{2}\right)$ fits best. The 2D-LIF band at $600 \mathrm{~cm}^{-1}$ can be assigned to the totally-symmetric level $14_{1} 20_{1} m_{3(+)}$ - this must arise from FC activity, which is consistent with its similar profile and excitation position to the $14_{2}$ and $14_{1} m_{6(-)}$ bands.

There are various assignments for the emission band at $703 \mathrm{~cm}^{-1}$. A number of these can be excluded on the grounds that they do not fit the position of an expected $\Delta(v, m)=0$ band, or are not expected to have significant FC activity: $20_{3} m_{7}, 18_{1} m_{6( \pm)}, 20_{4} m_{5}, 30_{2} m_{5}$ and $17_{1} m_{2}$. Our favoured candidates for the emission are $14_{1} 2 O_{2} m_{1}$ and $14_{1} 20_{2} m_{2}$. Although the former is the best fit on the grounds of expected wavenumber, we cannot exclude the latter since there is significant lowering of some of the $m=2$ levels in the $S_{1}$ state arising from vibtor interactions. ${ }^{16}$

Indeed assignment of the $(397,703) \mathrm{cm}^{-1}$ band to $\left(14^{1} 20^{2} \mathrm{~m}^{1}, 14_{1} 20_{2} m_{1}\right)$ would not be consistent with the energy level diagrams presented in $\mathrm{GL}^{16}$ which suggests the upper level is above $420 \mathrm{~cm}^{-1}$ and so would not be expected at this excitation position. On the other hand, an assignment to $\left(14^{1} 20^{2} \mathrm{~m}^{2}\right.$, $14_{1} 2 \mathrm{O}_{2} m_{2}$ ) would be consistent with that work, if it is assumed there were vibtor interactions in the $S_{0}$ state that pushed the $14_{1} 20_{2} m_{2}$ level down. The $14^{1} 20^{2} m^{2}$ level would have to gain intensity from interacting with $29^{1} \mathrm{~m}^{1}$, which both have $e^{\prime}$ symmetry. Indeed, the 2D-LIF spectrum in Figure 9 shows weak $703 \mathrm{~cm}^{-1}$ emission across the $29^{1} \mathrm{~m}^{1}$ excitation region. We currently favour the assignment of the more intense part of the $703 \mathrm{~cm}^{-1}$ band to emission to $14^{1} 20^{2} \mathrm{~m}^{2} \ldots 29^{1} \mathrm{~m}^{1}$, with the weaker part to $29^{1} m^{1} \ldots 14^{1} 20^{2} m^{2}$. 
We do, however, wish to comment that if further interactions to those considered in Ref. 16 occurred in the $S_{1}$ state, then this may cause the $\left(14^{1} 20^{2} m^{1}, 14_{1} 20_{2} m_{1}\right)$ to become a viable assignment on energetic grounds; however, it would still be necessary to explain how the upper state gained intensity. An interaction with $14^{2} \mathrm{~m}^{1}$ is symmetry allowed, but the usual anharmonic and torsional coupling terms would not lead to such coupling, and so it would be necessary to invoke either an indirect coupling route (and it is unclear what this would be), Coriolis-type coupling via the involvement of rotations, or to invoke a new coupling term, possibly arising from the distortion of the torsional potential as a result of the angular variation of the interaction with the vibrational motion. Interestingly, we also see emission at $\sim 700 \mathrm{~cm}^{-1}$ when exciting at $365 \mathrm{~cm}^{-1}$, Figure 10, which could suggest a $\left(14^{1} 20^{1} m^{4}, 14_{1} 20_{2} m_{1}\right)$ symmetry-allowed assignment, or the less-likely $\left(14^{1} 20^{1} m^{4}, 14_{1} 20_{2} m_{2}\right)$, which would have to be vibronically induced and also involve $\Delta m=6$. There is also weak activity at $(412,703) \mathrm{cm}^{-1}$, which could be assigned to the symmetry-allowed $\Delta m=3$ transition $\left(29^{1} \mathrm{~m}^{2}\right.$, $\left.14_{1} 20_{2} m_{1}\right)$. We also note that there is some evidence for activity of $14^{1} 20^{2} \mathrm{~m}^{1}$ in absorption from the ZEKE spectra published in Ref. 15, where a weak band is seen at $572 \mathrm{~cm}^{-1}$ when exciting around the $14^{2}$ position (in the absence of further shifts from vibtor interactions, this band would be too low in wavenumber to arise from $14^{1} 20^{2} \mathrm{~m}^{2}$ ). Overall, however, the rationale for any such activity of the $14^{1} 20^{2} m^{1}$ activity is elusive. If the corresponding $m=1$ and $m=2$ levels were degenerate in $S_{1}$, this might also explain the observations if there were different transition intensities for these. As such, we cannot firmly decide on the complete assignment of the $703 \mathrm{~cm}^{-1}$ band, but have concluded it arises from emission to $14_{1} 20_{2} m_{x}(x=1$ and/or 2$)$ level, with the corresponding $S_{1}$ levels interacting with either $14^{2} m^{1}$ or $29^{1} m^{1}$, as discussed.

Finally, the $(399,713) \mathrm{cm}^{-1}$ band can be assigned to $\left(29^{1}, 20_{2} 29_{1}\right)$, and the band at $(409,743) \mathrm{cm}^{-1}$ is straightforwardly assigned as $\left(11^{1}, 11_{1} 20_{2}\right)$.

3.4.3.4. Comments on bands $750-920 \mathrm{~cm}^{-1}$. We now return to the region of the 2D-LIF spectrum shown in Figure $8(a)$. The weak band at $(398,766) \mathrm{cm}^{-1}$ is aligned with the $14_{2}$ band and the best assignment we have found is $\left(14^{2}, 14_{1} 30_{1} m_{3(-)}\right)$, while the band at $(399,777) \mathrm{cm}^{-1}$ is located at the position of $29^{1}$ and so is plausibly $\left(29^{1} \mathrm{~m}^{0}, 10_{1} m_{3(+)}\right)$. The $2 \mathrm{D}$-LIF band at $800 \mathrm{~cm}^{-1}$ appears to arise from FC activity and have a similar profile and position to the $14_{2}$ and $14_{1} m_{6(-)}$ bands, a reasonable assignment involving totally-symmetric levels is $\left(14^{2} m^{0}, 14_{1} 19_{1} m_{3(+)}\right)$

Both $\left(14^{2}, 9_{1}\right)$ and $\left(11^{1}, 9^{1}\right)$ bands, and possibly a very weak $\left(29^{1}, 9_{1}\right)$ band, are seen in the spectrum. Interestingly, there is a clear $\left(29^{1}, 29_{2}\right)$ band, even though it is symmetry forbidden and so suggesting 
$\mathrm{HT}$ activity. There is also a $\left(11^{1}, 29_{2}\right)$ band, which is very weak, even though it is symmetry allowed; there is, however, no definitive evidence of a $\left(14^{2}, 29_{2}\right)$ band. At $(399,879) \mathrm{cm}^{-1}$ is the clear $\left(29^{1}\right.$, $\left.11_{1} 29_{1}\right)$ band, and there also seems to be a very faint $\left(11^{1}, 11_{1} 29_{1}\right)$ band, which is easier to discern in the DF spectrum (Figure 4); this could be indicative of very weak HT activity between $11^{1}$ and $29^{1}$, with the band intensity enhanced because of the excitation at the $11^{1}$ position. There is also a significant $\left(11^{1}, 11_{2}\right)$ band, which may have a much weaker $\left(14^{2}, 11_{2}\right)$ FC-active counterpart, but the latter could also arise from a contribution from $2 \mathrm{O}_{2} 14_{1} m_{6(-)}$.

3.4.3.5 Comments on bands $\mathbf{9 2 0}-\mathbf{1 1 5 0} \mathrm{cm}^{-1}$. In Figure 11 we show an expanded view of the 2D-LIF spectrum covering the range $(390-415,940-1150) \mathrm{cm}^{-1}$. A very weak band at $\left(399,943 \mathrm{~cm}^{-1}\right)$ seems likely to be associated with $11_{1}$ and the level that gives rise to the $489 \mathrm{~cm}^{-1}$ band - see Section 3.4.3.2 - and hence be $\left(20^{2} \mathrm{~m}^{6(+)}, 11_{1} 20_{2} m_{6(+)}\right)$. A very weak band at $(398,990) \mathrm{cm}^{-1}$ is potentially the $\left(14^{2} \mathrm{~m}^{1}\right.$, $\left.14_{1} 18_{1} m_{4}\right)$ band.

The symmetry-forbidden $\left(29^{1}, 28_{1} 2_{1}\right)$ band is present at $(399,1062) \mathrm{cm}^{-1}$, and ties in with the $29_{1}, 28_{1}$ and $29_{2}$ activity at this excitation position. The band at $(398,1071) \mathrm{cm}^{-1}$ can be associated with emission to $11_{1} 14_{1} m_{6(-)}$ as the band profile closely matches that of the $(398,618) \mathrm{cm}^{-1}$ band associated with emission to $14_{1} m_{6(-)}$, discussed in Section 3.4.2.

There are two bands associated with emission at $1091 \mathrm{~cm}^{-1}$, appearing when exciting $29^{1}$ and $11^{1}$ positions. The $(399,1091) \mathrm{cm}^{-1}$ band can be straightforwardly assigned as $\left(29^{1}, 11_{1} 28_{1}\right)$, but since we do not see a significant $\left(11^{1}, 28_{1}\right)$ band and also as the $\left(11^{1}, 11_{1} 29_{1}\right)$ bands were weak, this suggests that the $(409,1091) \mathrm{cm}^{-1}$ band is unlikely to be $\left(11^{1}, 11_{1} 28_{1}\right)$. Our favoured assignment is to $11_{1} 18_{1} 20_{1}$. Particularly as the band profiles look to be different, and the centres of the bands are at slightly different emission wavenumbers. Although there is only the slightest trace of the $18_{1} 20_{1}$ band that is expected to appear at $\sim(409,640) \mathrm{cm}^{-1}$, it is likely that the $\left(11^{1}, 11_{1} 18_{1} 20_{1}\right)$ band is enhanced by the $11_{1}$ character when exciting at $11^{1}$, as seen for many bands in the DF spectra in Figures 4 and 5.

There is a very weak band at $(397,1110) \mathrm{cm}^{-1}$ that is assignable to $\left(14^{2}, 14_{2} 20_{2}\right)$; an exceptionally weak band at $(409,1119) \mathrm{cm}^{-1}$ that is assignable as $\left(11^{1}, 11_{1} 19_{2}\right)$ and a weak band at $(398,1129) \mathrm{cm}^{-1}$ that is assignable as $\left(14^{2}, 9_{1} 20_{2}\right)$.

3.4.3.6 Bands to higher wavenumber. In Figure 12 we show the region of the 2D-LIF spectrum associated with a higher excitation wavenumber. Several very weak bands appear, whose assignments 
can be deduced as follows, based on the agreement of their positions with both the expected excitation and emission wavenumbers.

The band at $(438,513) \mathrm{cm}^{-1}$ is assigned to $\left(18^{1} \mathrm{~m}^{2}, 18_{1} \mathrm{~m}_{2}\right)$. There are two plausible assignments of the band at $(451,452) \mathrm{cm}^{-1},\left(30^{1} \mathrm{~m}^{5}, 30_{1} \mathrm{~m}_{5}\right)$ and $\left(\mathrm{m}^{9 \pm}, m_{9 \pm}\right)$, but a definitive assignment is not possible at this time. The band at $(463,544) \mathrm{cm}^{-1}$ seems likely to be $\left(18^{1} \mathrm{~m}^{3(-)}, 18_{1} m_{3(-)}\right)$. The feature at $(482,668)$ $\mathrm{cm}^{-1}$ was assigned to $\left(19^{2}, 19_{2}\right)$, by GL, ${ }^{16}$ with which we concur. They saw a separation of the $m=0$ and $m=1$ components, which is just about discernible in expanded views of our image. Finally, the band at $(494,668) \mathrm{cm}^{-1}$ is assigned to the $\Delta(v, m)=0$ transition, $\left(19^{1} 20^{2} \mathrm{~m}^{3(-)}, 19_{1} 20_{2} m_{3(-)}\right)$.

To higher wavenumber in the DF spectra (Figures 4 and 5) we see some other features. The DF band at $1071 \mathrm{~cm}^{-1}$ (Figure 4 ) is assigned as $11_{1} 14_{1} m_{6(-)}$ with its band shape and location consistent with that of the $618 \mathrm{~cm}^{-1}$ band assigned as emission to $14_{1} m_{6(-)}$. In Figure 4, we see the $18_{2}$ band at $1000 \mathrm{~cm}^{-1}$, with transitions involving the $D_{18}$ overtone being the subject of our recent paper using ZEKE and 2DLIF spectroscopy. ${ }^{29}$ The three intense DF bands at $1215 \mathrm{~cm}^{-1}, 1228 \mathrm{~cm}^{-1}$ and $1241 \mathrm{~cm}^{-1}$ (Figure 5) can be assigned as $6_{1}, 13_{1} 14_{1}$ and $5_{1}$, respectively, with the middle band providing a value for $D_{13}$ in the $S_{0}$ state, which is included in Table 1. Lastly, we mention the $11_{3}$ DF band, which appears when exciting via $11_{1}$, concomitant with the strong enhancements of $11_{1}$ and $11_{2}$ via the $11^{1}$ excitation (Figures 4 and 5). The $11^{n}$ progression seems very harmonic - consistent with at most a weak interaction of $11^{1}$ with $14^{2}$.

\section{Final remarks and conclusions}

We have presented a wide region of the 2D-LIF spectrum, and separately-recorded associated DF spectra, across excitation wavenumbers $360-430 \mathrm{~cm}^{-1}$. These have revealed a wealth of underlying structure and the activity has provided insight into the activity of different $S_{1}$ ZOSs. The results are significant since they reveal extensive vibration-torsion activity. We have seen the fundamentals $29_{1}$, $28_{1}, 11_{1}, 10_{1}, 9_{1}, 7_{1}, 6_{1}$ and $5_{1}$ giving gas-phase values for the corresponding vibrations, and also for the $S_{0} D_{13}$ mode via the $13_{1} 14_{1}$ combination band, and these are all included in Table 1 . 
Comparison of the different DF spectra in Figures 4 and 5 show that the changing relative intensities of the bands across the spectra are a powerful confirmation of many of their assignments. The DF bands from $790-890 \mathrm{~cm}^{-1}$ are a case in point (see Figure 4) where the changing activities are very profound: the $9_{1}$ band dominates the spectrum via the origin, but is extremely weak via $29^{1}$; also there appears to be little correspondence in the profiles of the $9_{1}$ and $29_{2}$ bands in the $S_{0}$ state. The fact that $29_{2}$ is very weak via $14^{2}$ suggests that the $29_{2}$ band seen when exciting via $29^{1}$ is likely HT activity, rather than a result of any $14^{2} \ldots 29^{1}$ interaction or overlap in band profiles.

Indeed, we find little evidence that there is any significant interaction between the $14^{2}, 29^{1}$ and $11^{1}$ levels. The 2D-LIF and DF spectra show evidence of weak cross activity between $11^{1}$ and $14^{2}$, but this could simply be FC activity. We have previously concluded that these levels are close to coincident in the $p$ CIFB molecule, ${ }^{46}$ but that there was no clear evidence of interaction from the ZEKE spectra reported therein. In $p \mathrm{FT}$, we have seen herein that there is also little evidence for any significant interaction between these two levels, which is in agreement with the results of our ZEKE study. ${ }^{15}$ Symmetry suggests that cross activity between the $29^{1}$ and $14^{2}$ levels could occur as a result of HT coupling, which would be strongly dependent on the relative energies of the electronic states involved, and so vary from molecule to molecule; another possibility is rotation-induced coupling such as suggested for the $14^{2} m^{0} \ldots 14^{1} m^{6(-)}$ interaction. ${ }^{16,45}$ This would be a Coriolis-like interaction, but with its efficiency also expected to be strongly dependent on rotational levels coming in and out of resonance, and would be expected to be more prevalent at higher temperatures. Since we are working under jet-cooled conditions, the requirement for resonance would be more pronounced. However, currently there is little evidence from the spectra to infer that there is any significant cross activity between the $29^{1}$ and $14^{2}$ levels, which therefore excludes significant coupling of these levels. As a consequence, we conclude that each of the three states, $11^{1}, 29^{1}$ and $14^{2}$ are inherently 'bright', again in agreement with conclusions we have made in other work on $p \mathrm{FT}$ and related molecules. , $^{815,33,46}$

We have previously discussed in depth ${ }^{29}$ a feature that appears at $\mathrm{S}_{1}+845 \mathrm{~cm}^{-1}$ and the interactions there were isolated to the $18^{2} \mathrm{~m}^{1}$ level, which allowed couplings to vibtor combinations involving $m=$ 2. In so-doing, the mechanism opens up couplings between vibrations of different symmetry; such is also included in the vibtor coupling of the three lowest-wavenumber vibrations included in the model of GL. ${ }^{16}$ In our recent study of the $\mathrm{S}_{1} 0^{0}+845 \mathrm{~cm}^{-1}$ band, ${ }^{29}$ the $18^{2} \mathrm{~m}^{1}$ ZOB state was concluded as interacting with combinations formed from the $18^{1} \mathrm{~m}^{2}$ vibtor level with each of the $14^{2}, 29^{1}$ and $11^{1}$ levels, which are part of the present work. Pairwise interactions with the ZOB state occurred and it was possible that weak interactions between the so-formed eigenstates was also present, although a 
clear interpretation was hindered by further dissipative IVR processes. Thus, that work would be consistent with the at most weak interactions between the $14^{2}, 29^{1}$ and $11^{1}$ ZOSs concluded in the present work, taking on board that the ZOS separations and couplings will not be identical to those in the combination bands.

A significant interaction does occur between $14^{2}$ and $14^{1} \mathrm{~m}^{6(-)}$, which has been suggested ${ }^{16,45}$ as occurring via vibration-torsion-rotational coupling; additionally we have concluded that there is a possible interaction between $14^{2} \mathrm{~m}^{0}$ and $20^{2} \mathrm{~m}^{6(+)}$. Frustratingly, the appearance of the $703 \mathrm{~cm}^{-1}$ emission band suggests that there may be an interaction between $14^{2} \mathrm{~m}^{1}$ and $14^{1} 20^{2} \mathrm{~m}^{1}$, but the mechanism for this is unclear, but could be vibrational-rotational (Coriolis) in origin, but we currently favour the $14^{1} 20^{2} \mathrm{~m}^{2} \ldots 29^{1} \mathrm{~m}^{1}$ interaction. Moreover, we particularly highlight the appearance of many vibtor bands that have activity involving $14_{1}$ when exciting via $14^{2}$, with a number of these also involving combinations with $20_{1}$, such as $14_{1} 20_{1} m_{2}$ at $576 \mathrm{~cm}^{-1}, 14_{1} 20_{1} m_{3(+)}$ at $600 \mathrm{~cm}^{-1} 14_{1} 20_{1} m_{4}$ at 634 $\mathrm{cm}^{-1}, 14_{1} 20_{2} m_{1}$ at $703 \mathrm{~cm}^{-1}$, as well as $14_{1} 19_{1} m_{3(+)}$ at $800 \mathrm{~cm}^{-1}$. We explicitly note that the transitions involving $m_{1}$ and $m_{2}$ and $m_{4}$ must originate from $14^{2} m^{1}$, while transitions involving $m_{0}, m_{3(+)}$ and $m_{6( \pm)}$ must originate from $14^{2} m^{0}$. Further, we have seen that the $29_{1} m_{2}$ level is active when exciting via $11^{1}$ and suggests an interaction between the $29^{1} \mathrm{~m}^{2}$ and $11^{1} \mathrm{~m}^{1}$ levels. Overall, there is compelling evidence for the involvement of the $m^{1}$ torsional level in promoting activity through vibration-torsion coupling mechanisms. In principle, if the band centres can be precisely determined, then quantitative information on the interactions between the various levels can be extracted from the spectra, as done by GL recently, ${ }^{16}$ although all pertinent levels would ideally be observed. It is also possible to calculate the interactions using quantum chemistry, but this would require reliable anharmonic couplings and/or van der Waals interactions to be calculated. Both of these are challenging, particularly in the $\mathrm{S}_{1}$ state, where the out-of-plane vibrations prove to be challenging. ${ }^{15,28}$

That we see significant couplings at these very low wavenumbers is, of course, unusual. In Ref. 33 we have explicitly looked at the density of states, comparing $p \mathrm{CIFB}, p \mathrm{FT}, p \mathrm{DFB}$ and $p \mathrm{Xyl}$. There we saw that there was a very erratic build-up in the density of states of 'pure' vibrational levels that did not differ too much for each of the molecules at $<1000 \mathrm{~cm}^{-1}$; however, there was a dramatic increase in the DOS once vibtor levels were included, but the build-up is still erratic at very low wavenumbers. It is becoming indisputable that there is clear evidence for the methyl rotor having a dramatic direct impact on the make-up of the energy levels of molecules, and that this impacts on the couplings between these and so, as a consequence, on photophysical and photochemical behaviour. 


\section{Acknowledgements}

We are grateful to the EPSRC for funding (grant EP/L021366/1) and to the High Performance Computer resource at the University of Nottingham. The EPSRC and the University of Nottingham are thanked for a studentship to D.J.K. We note that Lewis Warner (Royal Society of Chemistry Summer Bursary student) helped in the recording of several dispersed fluorescence spectra. We acknowledge extremely useful discussions with Warren Lawrance and Jason Gascooke (Flinders, Adelaide) and thank them for sharing unpublished data and clarifying some aspects of vibration-torsional coupling.

\section{Conflicts of interest}

There are no conflicts to declare. 


\section{Reference}

${ }^{1}$ S. M. Bellm, P. T. Whiteside, and K. L. Reid, J. Phys. Chem. A 107, 7373 (2003).

${ }^{2}$ P. T. Whiteside, A. K. King, and K. L. Reid, J. Chem. Phys. 123, 204316 (2005).

${ }^{3}$ P. T. Whiteside, A. K. King, J. A. Davies, K. L. Reid, M. Towrie, and P. Matousek, J. Chem. Phys. 123, 204317 (2005).

${ }^{4}$ C. J. Hammond, K. L. Reid, and K. L. Ronayne, J. Chem. Phys. 124, 201102 (2006).

${ }^{5}$ J. A. Davies, A. M. Green, and K. L. Reid, Phys. Chem. Chem. Phys. 12, 9872 (2010).

${ }^{6}$ J. A. Davies, A. M. Green, A. M. Gardner, C. D. Withers, T. G. Wright, and K. L. Reid, Phys. Chem. Chem. Phys. 16, 430 (2014).

${ }^{7}$ A. M. Gardner, A. M. Green, V. M. Tamé-Reyes, K. L. Reid, J. A. Davies, V. H. K. Parkes, and T. G. Wright, J. Chem. Phys. 140, 114308 (2014).

${ }^{8}$ A. M. Gardner, A. M. Green, V. M. Tamé-Reyes, V. H. K. Wilton, and T. G. Wright, J. Chem. Phys. 138, 134303 (2013).

${ }^{9}$ E. A. Virgo, J. R. Gascooke, and W. D. Lawrance, J. Chem. Phys. 140, 154310 (2014).

10 J. R. Gascooke, E. A. Virgo, and W. D. Lawrance, J. Chem. Phys. 142, 024315 (2015).

${ }^{11}$ J. R. Gascooke, E. A. Virgo, and W. D. Lawrance, J. Chem. Phys. 143, 044313 (2015).

12 J. R. Gascooke and W. D. Lawrance, J. Molec. Spec. 318, 53 (2015).

${ }^{13}$ J. R. Gascooke and W. D. Lawrance, Eur. Phys. J. D 71, 287 (2017).

${ }^{14}$ V. L. Ayles, C. J. Hammond, D. E. Bergeron, O. J. Richards, and T. G. Wright, J. Chem. Phys. 126, 244304 (2007).

${ }^{15}$ A. M. Gardner, W. D. Tuttle, L. Whalley, A. Claydon, J. H. Carter, and T. G. Wright, J. Chem. Phys. 145,124307 (2016).

${ }^{16}$ J. R. Gascooke, L. D. Stuart, P. G. Sibley, and W. D. Lawrance, J. Chem. Phys. 149, 074301 (2018). This work contains considerable extra information in its supplementary information. Note, however, that the excitation wavenumber axis is incorrect in Figure S9.

${ }^{17}$ T. Cvitaš and J. M. Hollas, Molec. Phys. 20, 645 (1971).

${ }^{18}$ C. J. Seliskar, M. A. Leugers, M. Heaven, and J. L. Hardwick, J. Mol. Spect. 106, 330 (1984).

${ }^{19}$ K. Okuyama, N. Mikami, and M. Ito, J. Phys. Chem. 89, 5617 (1985).

${ }^{20}$ Y. M. Ha, I. S. Choi, and S. K. Lee, Bull. Kor. Chem. Soc. 19, 202 (1998).

${ }^{21}$ Z.-Q. Zhao and C. S. Parmenter, Mode Selective Chemistry (Kluwer, 1991) Eds. J. Jortner, R. D. Levine, and B. Pullman. Jerusalem Symp. Quant. Chem. Biochem. 24, 127 (1991).

${ }^{22}$ Z.-Q. Zhao, C. S. Parmenter, D. B. Moss, A. J. Bradley, A. E. W. Knight, and K. G. Owens, J. Chem. Phys. 96, 6362 (1992).

${ }^{23}$ Z.-Q. Zhao, PhD Thesis, Indiana University (1992). 
${ }^{24}$ Q. Ju, C. S. Parmenter, T. A. Stone, and Z.-Q. Zhao, Isr. J. Chem. 37, 379 (1997).

${ }^{25}$ S. Georgiev, T. Chakraborty, and H. J. Neusser, J. Phys. Chem. A 108, 3304 (2004).

${ }^{26}$ Y. Hu, X. Wang, and S. Yang, Chem. Phys 290, 233 (2003).

27 J. A. Davies and K. L. Reid, J. Chem. Phys., 2011, 135, 124305.

${ }^{28}$ W. D. Tuttle, A. M. Gardner, L. E. Whalley, and T. G. Wright, J. Chem. Phys. 146, 244310 (2017).

${ }^{29}$ A. M. Gardner, W. D. Tuttle, L. E. Whalley, and T. G. Wright, Chem. Sci. 9, 2270 (2018).

${ }^{30}$ Z.-Q. Zhao, PhD Thesis, Indiana University (1992).

${ }^{31}$ A. M. Gardner, W. D. Tuttle, P. Groner, and T. G. Wright, J. Chem. Phys. 146, 124308 (2017).

${ }^{32}$ W. D. Tuttle, A. M. Gardner, K. B. O’Regan, W. Malewicz, and T. G. Wright, J. Chem. Phys. 146, 124309 (2017).

${ }^{33}$ W. D. Tuttle, A. M. Gardner, L. E. Whalley, D. J. Kemp, and T. G. Wright, Phys. Chem. Chem. Phys. In Press. (2019) DOI: 10.1039/c8cp02757a

${ }^{34}$ E. B. Wilson, Jr, Phys. Rev. 45 (1934) 706.

${ }^{35}$ G. Varsányi, Assignments of the Vibrational Spectra of Seven Hundred Benzene Derivatives (Wiley, New York, 1974).

${ }^{36}$ R. S. Mulliken, J. Chem. Phys. 23, 1997 (1955).

${ }^{37}$ G. Herzberg, Molecular Spectra and Molecular Structure II. Infrared and Raman Spectra of Polyatomic Molecules (Krieger, Malabar, 1991).

${ }^{38}$ A. Andrejeva, A. M. Gardner, W. D. Tuttle, and T. G. Wright, J. Molec. Spectrosc. 321, 28 (2016).

${ }^{39}$ P. J. Breen, J. A. Warren, E. R. Bernstein, and J. I. Seeman, J. Chem. Phys. 87, 1917 (1987).

${ }^{40}$ I. Pugliesi, N. M. Tonge, and M. C. R. Cockett, J. Chem. Phys. 129, 104303 (2008).

${ }^{41}$ A. M. Gardner and T. G. Wright, J. Chem. Phys. 135, 114305 (2011).

${ }^{42}$ E. Fermi, Z. Phys. 71, 250 (1931).

${ }^{43}$ J. R. Gascooke and W. D. Lawrance, J. Chem. Phys., 2013, 138, 134302.

${ }^{44}$ N. T. Whetton and W. D. Lawrance, J. Phys. Chem., 1989, 93, 5377-5384.

45 J. R. Gascooke and W. D. Lawrance, unpublished work.

${ }^{46}$ D. J. Kemp, L. E. Whalley, W. D. Tuttle, A. M. Gardner, B. T. Speake, and T. G. Wright, Phys. Chem. Chem. Phys. 20, 12503 (2018). 
Table 1: Calculated and experimental vibrational wavenumbers $\left(\mathrm{cm}^{-1}\right)$ for the $\mathrm{S}_{0}, \mathrm{~S}_{1}$ and $\mathrm{D}_{0}{ }^{+}$electronic states of $p \mathrm{FT}^{\mathrm{a}}$

\begin{tabular}{|c|c|c|c|c|c|c|c|c|c|}
\hline & & \multicolumn{2}{|c|}{ Wilson/ Varsányi } & \multicolumn{2}{|c|}{$\mathrm{S}_{0}$} & \multicolumn{2}{|c|}{$\mathrm{S}_{1}$} & \multicolumn{2}{|c|}{$\mathrm{D}_{0}^{+}$} \\
\hline$D_{i}^{\mathrm{b}}$ & $\begin{array}{c}\text { Mulliken }{ }^{c} \\
\text { ' } C_{2 v}{ }^{\prime}\end{array}$ & Ref. 14 & Duschinsky $^{d}$ & B3LYPe & Expt $^{f}$ & B3LYPg & $\mathrm{Expt}^{\mathrm{h}}$ & B3LYPi & Expt $^{j}$ \\
\hline \multicolumn{10}{|c|}{$a_{1}^{\prime}$} \\
\hline$D_{1}$ & 1 & 2 & $2,7 a$ & 3103 & 3068 & 3130 & & 3116 & \\
\hline$D_{2}$ & 2 & $20 a$ & $13,20 a$ & 3071 & 3068 & 3105 & & 3101 & \\
\hline$D_{3}$ & 4 & $8 a$ & $9 a$ & 1598 & 1603 & 1528 & & 1628 & 1631 \\
\hline$D_{4}$ & 5 & $19 a$ & $18 a,(20 a)$ & 1499 & 1513 & 1432 & & 1454 & \\
\hline$D_{5}$ & 7 & $7 a$ & $1,7 a,(2,6 a)$ & 1209 & $1241^{k}$ & 1213 & 1230 & 1311 & 1332 \\
\hline$D_{6}$ & 8 & 13 & $\begin{array}{c}12,19 a, 20 a,(13 \\
18 a)\end{array}$ & 1192 & $1215^{k}$ & 1185 & 1194 & 1211 & 1230 \\
\hline$D_{7}$ & 9 & $9 a$ & $8 \mathrm{a}$ & 1145 & $1159^{k}$ & 1120 & & 1158 & 1170 \\
\hline$D_{8}$ & 10 & $18 a$ & $19 a, 12$ & 1005 & 1001 & 954 & & 969 & \\
\hline$D_{9}$ & 11 & 1 & $1,6 a,(7 a, 2)$ & 827 & $843^{\prime}$ & 805 & 797 & 811 & 824 \\
\hline$D_{10}$ & 12 & 12 & $20 a, 12,(19 a, 13)$ & 715 & $730^{k}$ & 700 & 711 & 710 & 721 \\
\hline$D_{11}$ & 13 & & & 446 & $453^{\prime}$ & 410 & $408^{1}$ & 437 & 440 \\
\hline \multicolumn{10}{|c|}{$a_{2}^{\prime}$} \\
\hline$D_{12}$ & 14 & $17 a$ & $17 a$ & 953 & 956 & 588 & 618 & 987 & 985 \\
\hline$D_{13}$ & 15 & $10 a$ & $10 a$ & 808 & 810 & 484 & 512 & 770 & 777 \\
\hline$D_{14}$ & 16 & $16 a$ & $16 a$ & 418 & $414^{\prime}$ & 172 & $199^{\prime}$ & 356 & 350 \\
\hline \multicolumn{10}{|c|}{$a_{2}^{\prime \prime}$} \\
\hline$D_{15}$ & 20 & 5 & $5,10 b$ & 931 & 929 & 706 & 678 & 998 & 1008 \\
\hline$D_{16}$ & 21 & $17 \mathrm{~b}$ & 17b,11,(16b) & 817 & 819 & 651 & 607 & 832 & 842 \\
\hline$D_{17}$ & 22 & 4 & $4,(10 b)$ & 698 & 695 & 538 & 509 & 671 & 685 \\
\hline$D_{18}$ & 23 & $16 \mathrm{~b}$ & $16 b, 11,(17 b)$ & 500 & $500^{k}$ & 468 & 426 & 488 & 499 \\
\hline$D_{19}$ & 24 & $10 \mathrm{~b}$ & $10 b,(4,5)$ & 330 & $334^{\prime}$ & 243 & $239^{1}$ & 266 & 271 \\
\hline$D_{20}$ & 25 & 11 & $16 b, 17 b, 11$ & 141 & $143^{\prime}$ & 110 & $104^{\prime}$ & 109 & 111 \\
\hline \multicolumn{10}{|c|}{$a_{1}^{\prime \prime}$} \\
\hline$D_{21}$ & 26 & $7 b$ & $20 \mathrm{~b}$ & 3102 & 3040 & 3126 & & 3115 & \\
\hline$D_{22}$ & 27 & $20 \mathrm{~b}$ & $7 b$ & 3071 & 3040 & 3100 & & 3101 & \\
\hline$D_{23}$ & 29 & $8 b$ & $9 b$ & 1586 & 1592 & 1427 & & 1383 & \\
\hline$D_{24}$ & 31 & $19 b$ & 18b,(19b,14,15) & 1395 & 1435 & 1315 & & 1470 & \\
\hline$D_{25}$ & 32 & 14 & 15,(14) & 1283 & 1300 & 935 & & 1301 & \\
\hline$D_{26}$ & 33 & 3 & $3,8 b$ & 1292 & 1321 & 1255 & & 1250 & \\
\hline$D_{27}$ & 34 & $18 \mathrm{~b}$ & $14,19 b$ & 1090 & 1099 & 1053 & & 1115 & \\
\hline$D_{28}$ & 36 & $6 b$ & $6 b,(8 b)$ & 633 & $640^{\prime}$ & 546 & 552 & 564 & 570 \\
\hline$D_{29}$ & 37 & $9 b$ & $8 b, 6 b,(3)$ & 414 & $424^{\prime}$ & 395 & $399^{\prime}$ & 412 & 416 \\
\hline$D_{30}$ & 38 & 15 & $19 b, 14,(18 b)$ & 298 & $307^{1}$ & 307 & $309^{1}$ & 313 & 320 \\
\hline
\end{tabular}


a Symmetries of the vibrations are given in the $G_{12}$ molecular symmetry group.

${ }^{\text {b }}$ See Ref. 38 for a discussion of these labels and to see mode diagrams.

' Used in Ref. 14 and related work - these are rather misleading labels, as the numbering included the vibrations of the methyl group, which cannot be described in $C_{2 v}$.

${ }^{d}$ These express the $S_{0}$ modes of $p F T$ in terms of those of benzene, using a generalised Duschinsky approach involving artificial isotopologues - see Ref. 38. Values outside parentheses have mixing coefficients $>0.2$ and are termed major contributions, with bolded values being dominant contributions (mixing coefficients $>0.5$ ). Those inside parentheses are minor contributions, and have values between 0.05 and 0.2 . If there is more than one contribution of each type, these are given in contribution order. Vibrations with a mixing coefficient $<0.05$ are ignored.

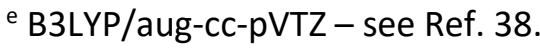

${ }^{f}$ From Ref. 38 unless otherwise noted. See that work for a discussion of the assignments and references to original papers.

g TD-B3LYP/aug-cc-pVTZ - see Ref. 28.

${ }^{h}$ From Ref. 28 unless otherwise noted.

' UB3LYP/aug-cc-pVTZ - see Ref. 28.

${ }^{j}$ From Refs. 14, 15 and 28

${ }^{\mathrm{k}}$ From present work.

' From Ref. 16 and consistent with Ref. 28 and present work. 
Table 2: Correspondence of the $C_{2 v}$ point group symmetry classes with those of the $G_{12}$ molecular symmetry group. Also indicated are the symmetries of the different pure torsional levels. ${ }^{a}$

\begin{tabular}{|l|l|l|}
\hline$C_{2 v}$ & $G_{12}$ & $m$ \\
\hline$a_{1}$ & $a_{1}{ }^{\prime}$ & $0,6(+)$ \\
\hline$a_{2}$ & $a_{2}{ }^{\prime}$ & $6(-)$ \\
\hline$b_{1}$ & $a_{2}^{\prime \prime}$ & $3(-)$ \\
\hline$b_{2}$ & $a_{1}^{\prime \prime}$ & $3(+)$ \\
\hline & $e^{\prime}$ & 2,4 \\
\hline & $e^{\prime \prime}$ & 1,5 \\
\hline
\end{tabular}

a Symmetries of vibtor levels can be obtained by combining the vibrational symmetry (in $G_{12}$ ) with those of the pure torsional level, using the direct product table of the isomorphic $D_{3 \mathrm{~h}}$ point group. Vibrational symmetries are given in Table 1. 
Figure 1

(a)

REMPI

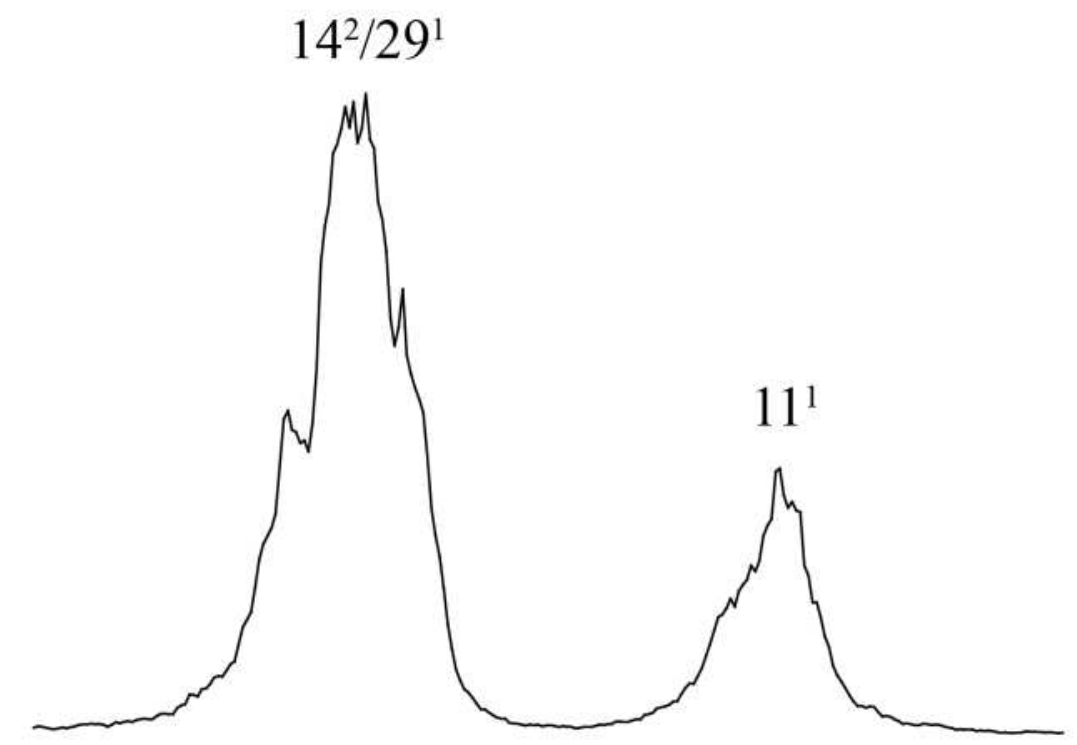

(b)

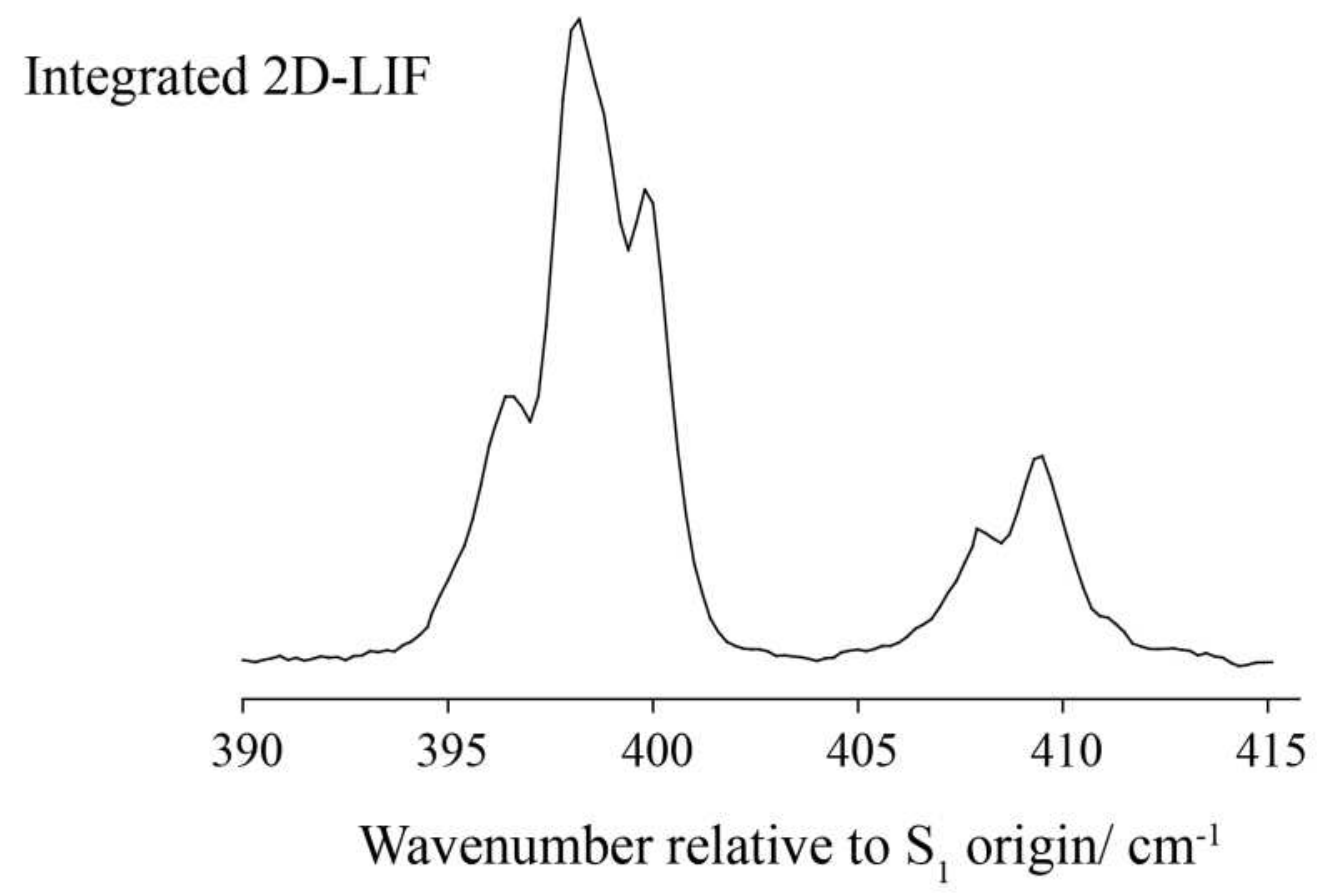


Figure 2

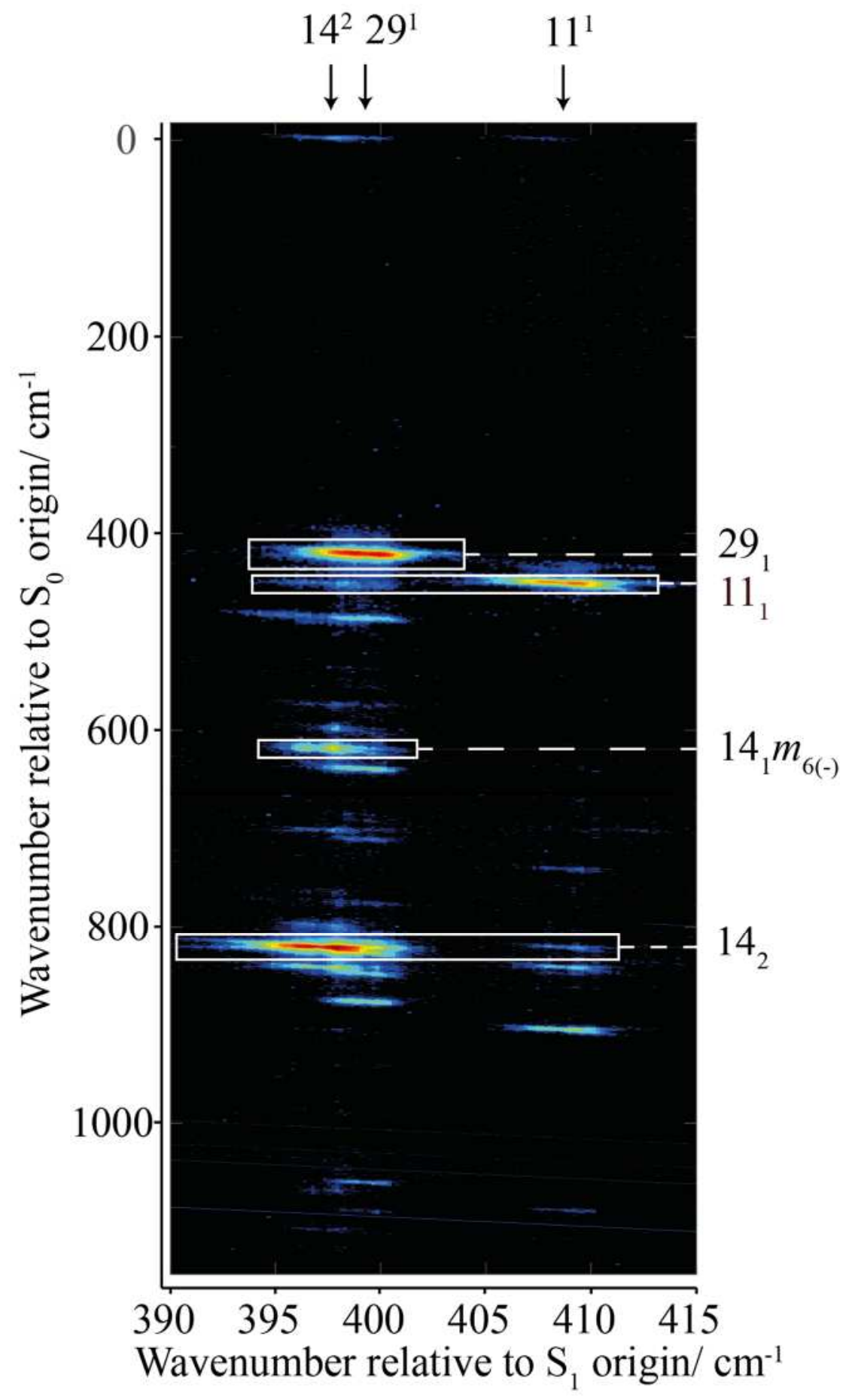


Figure 3

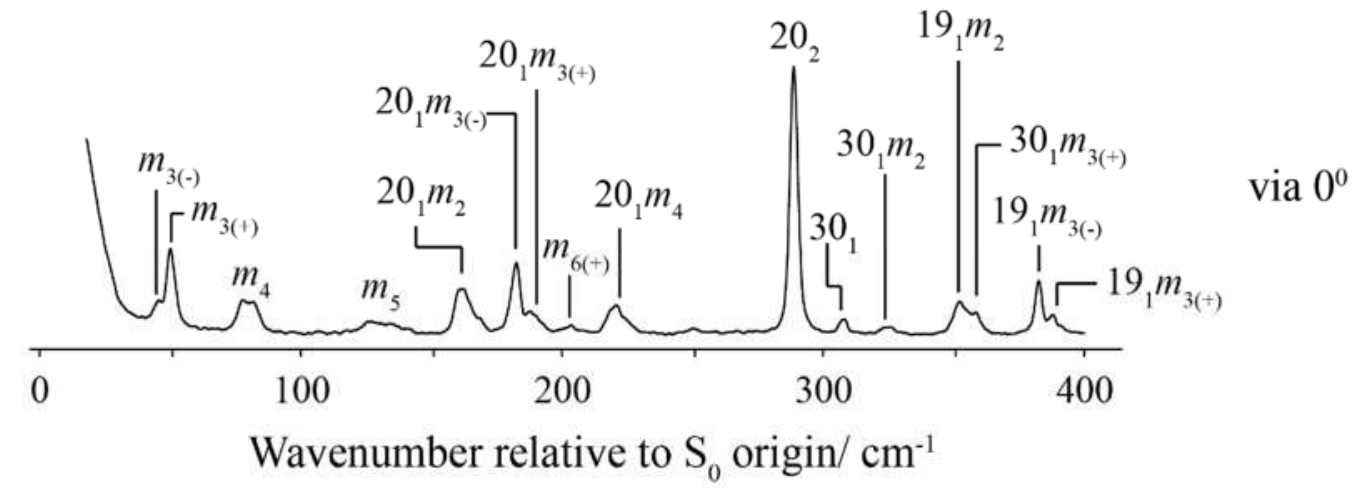


Figure 4

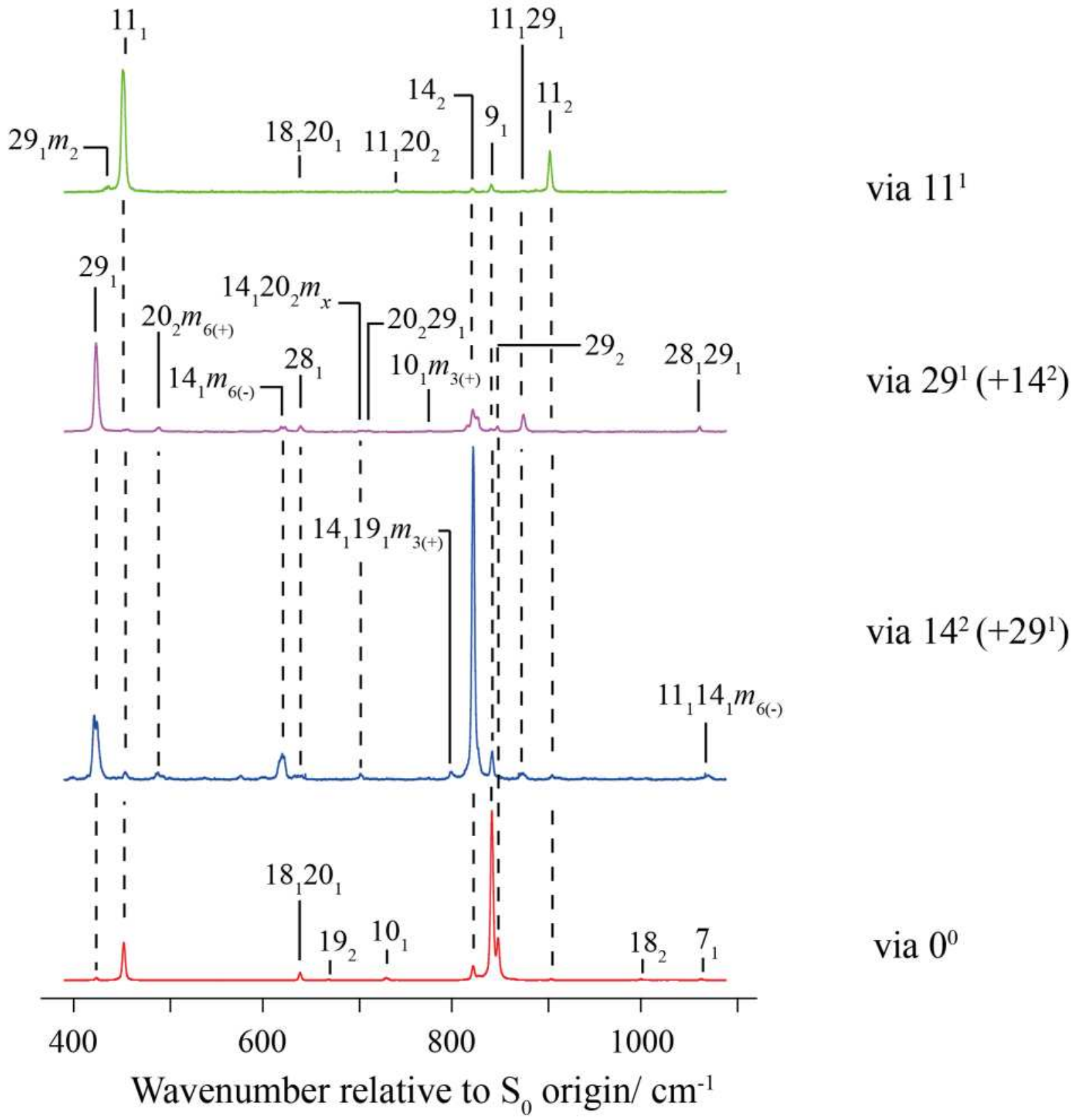


Figure 5

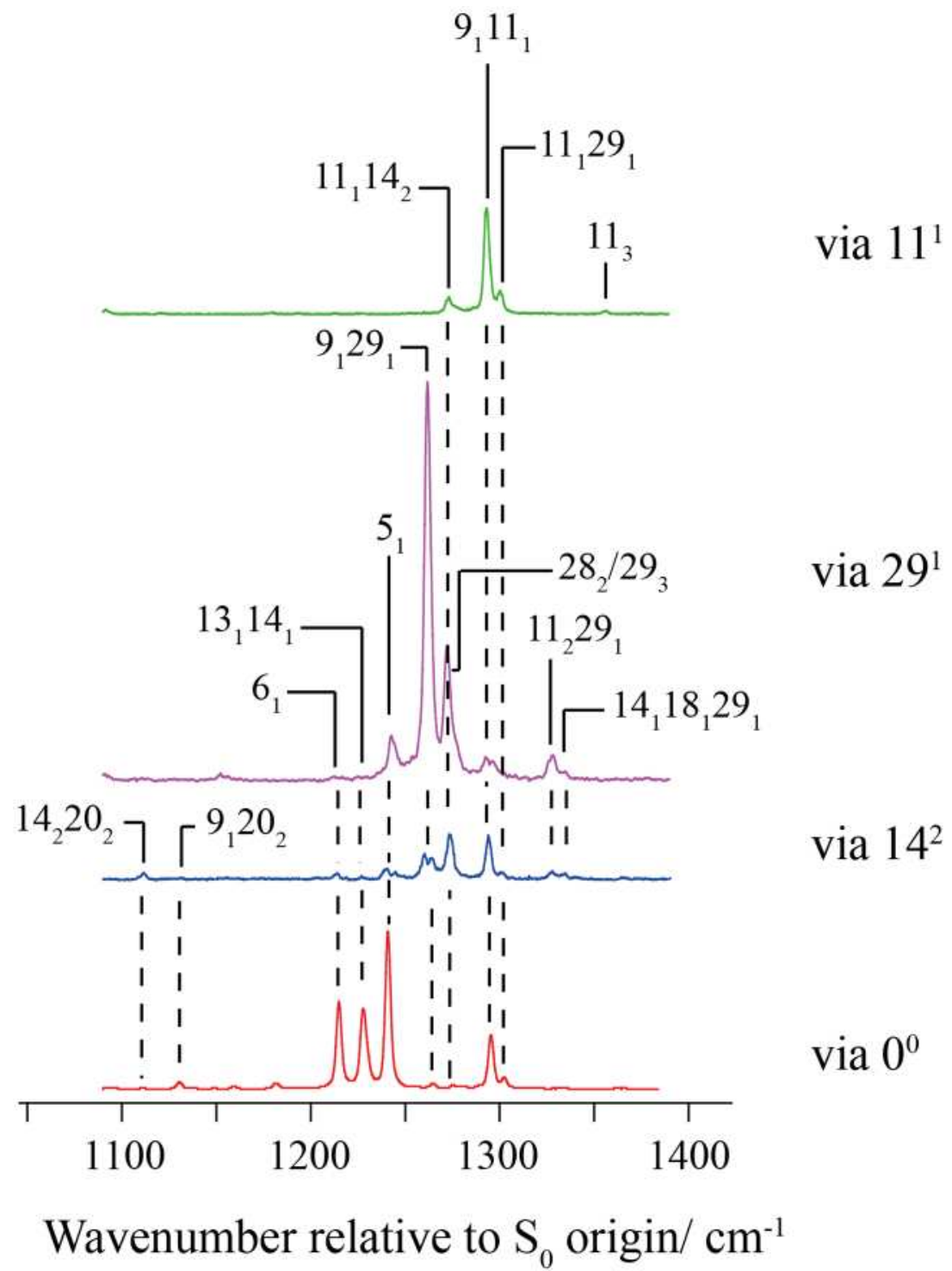


Figure 6

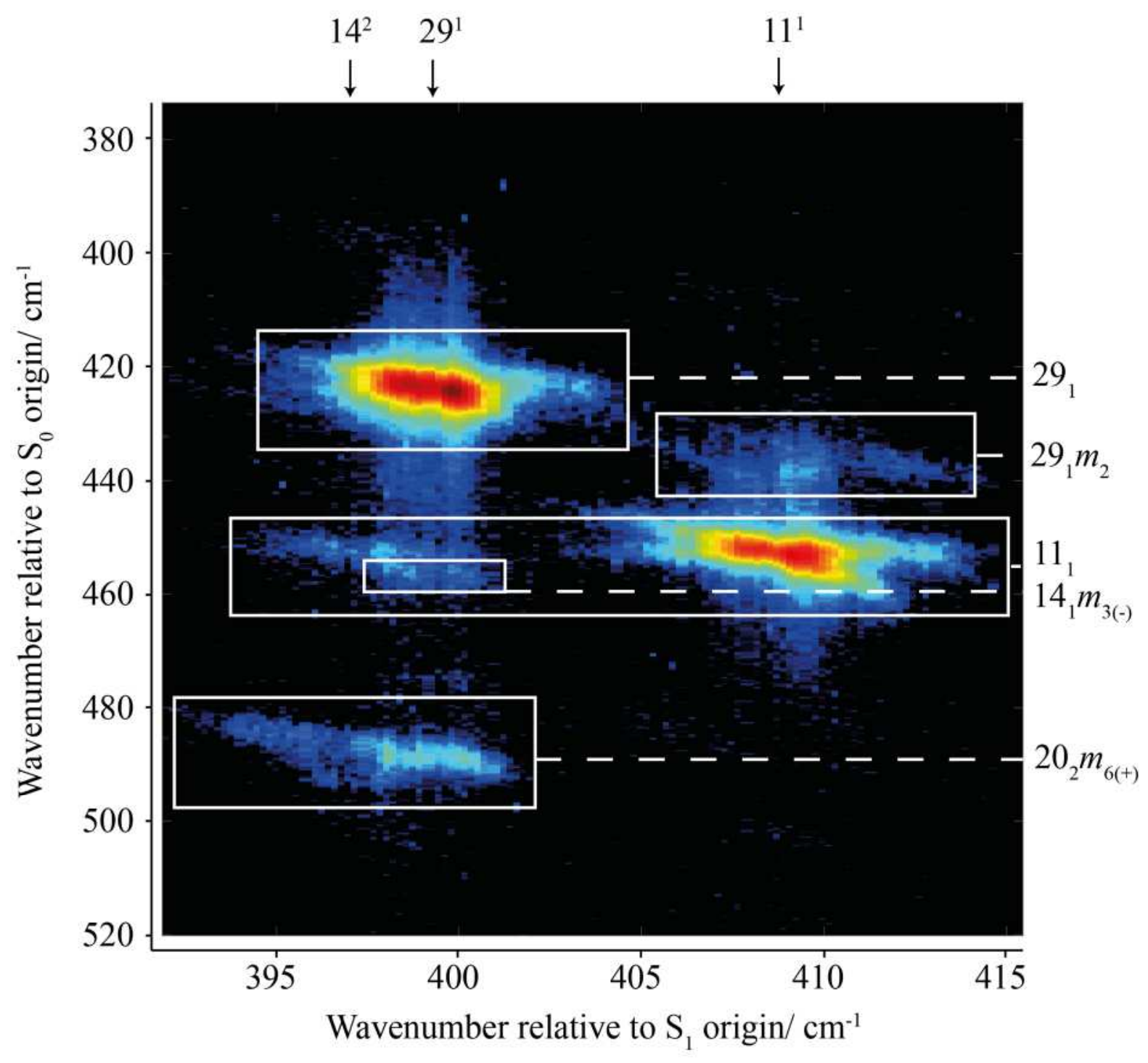


Figure 7

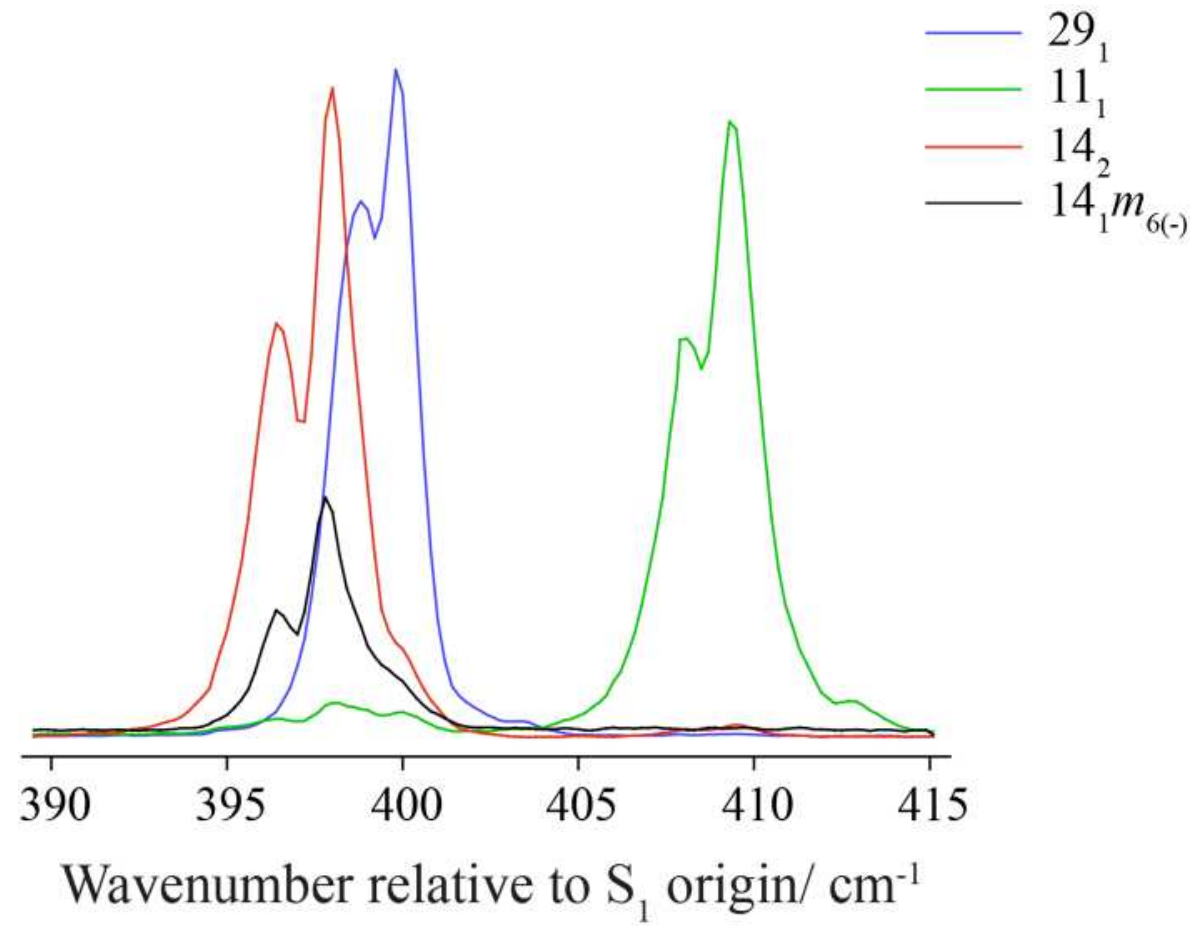


Figure 8

(a)

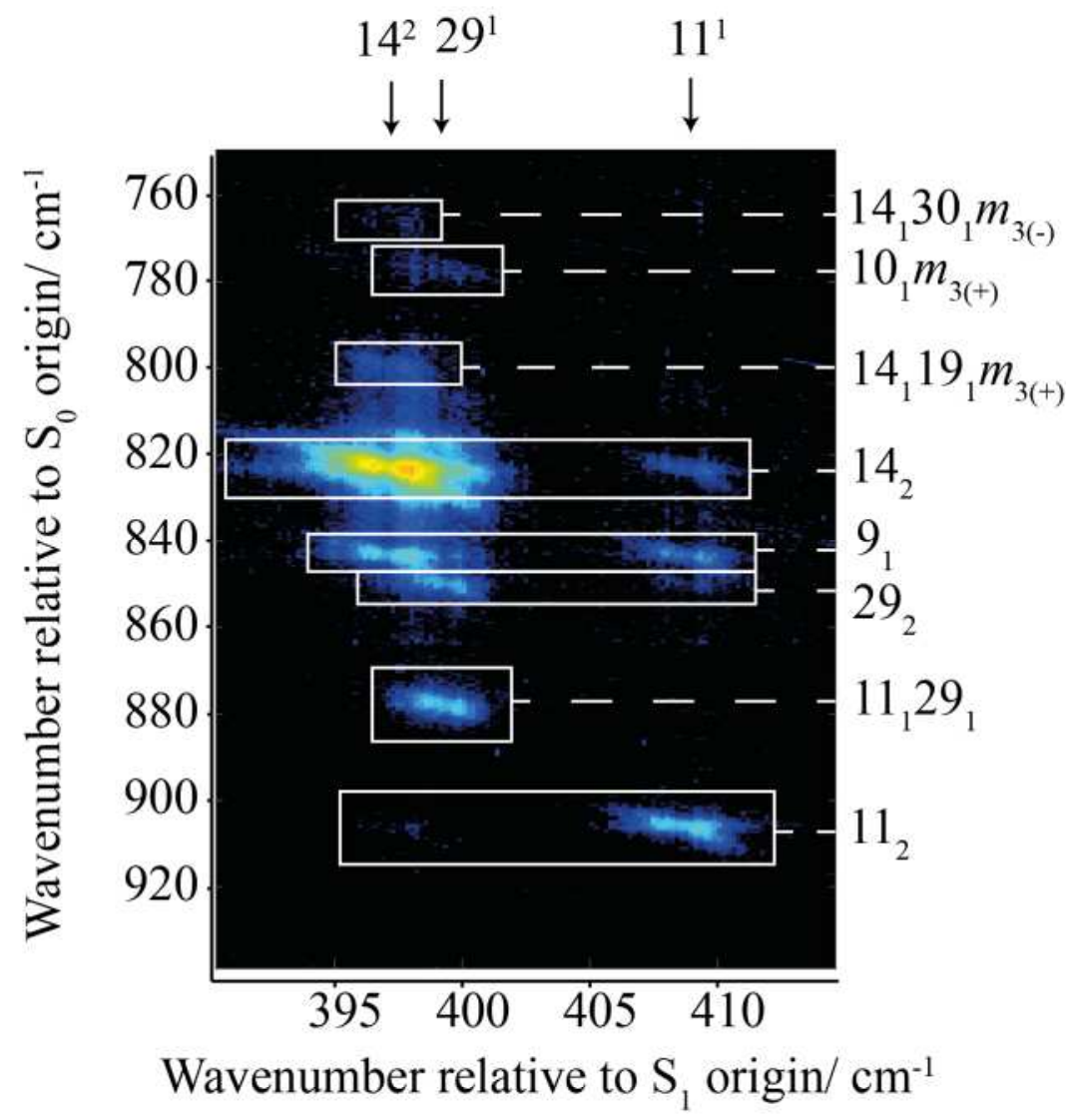

(b)

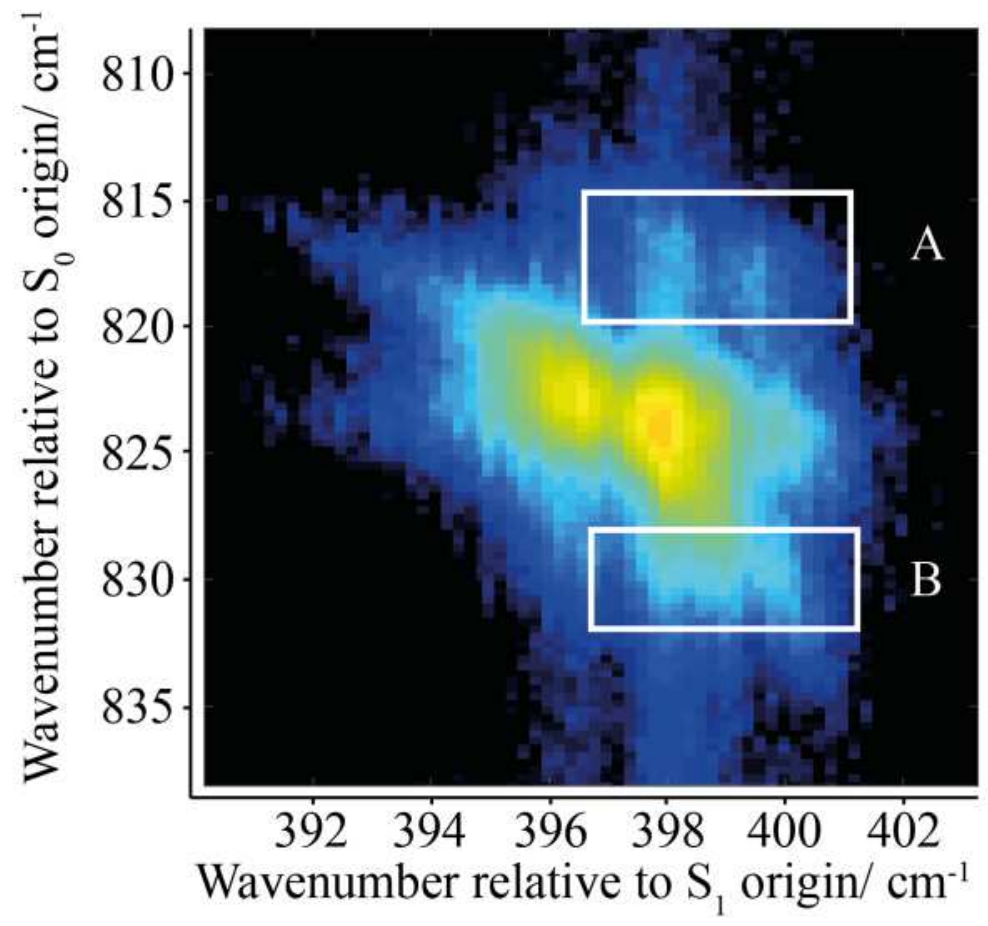


Figure 9

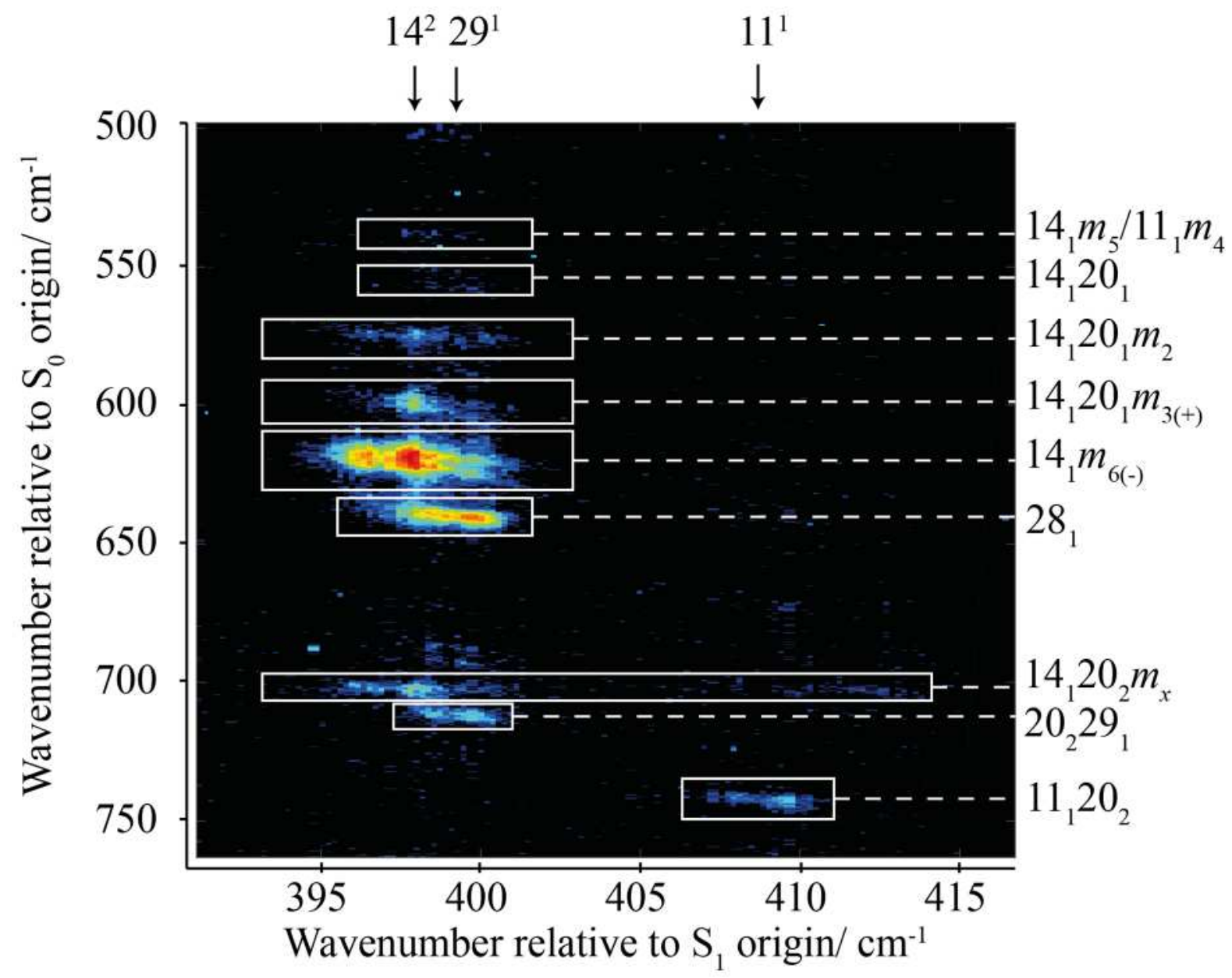


Figure 10

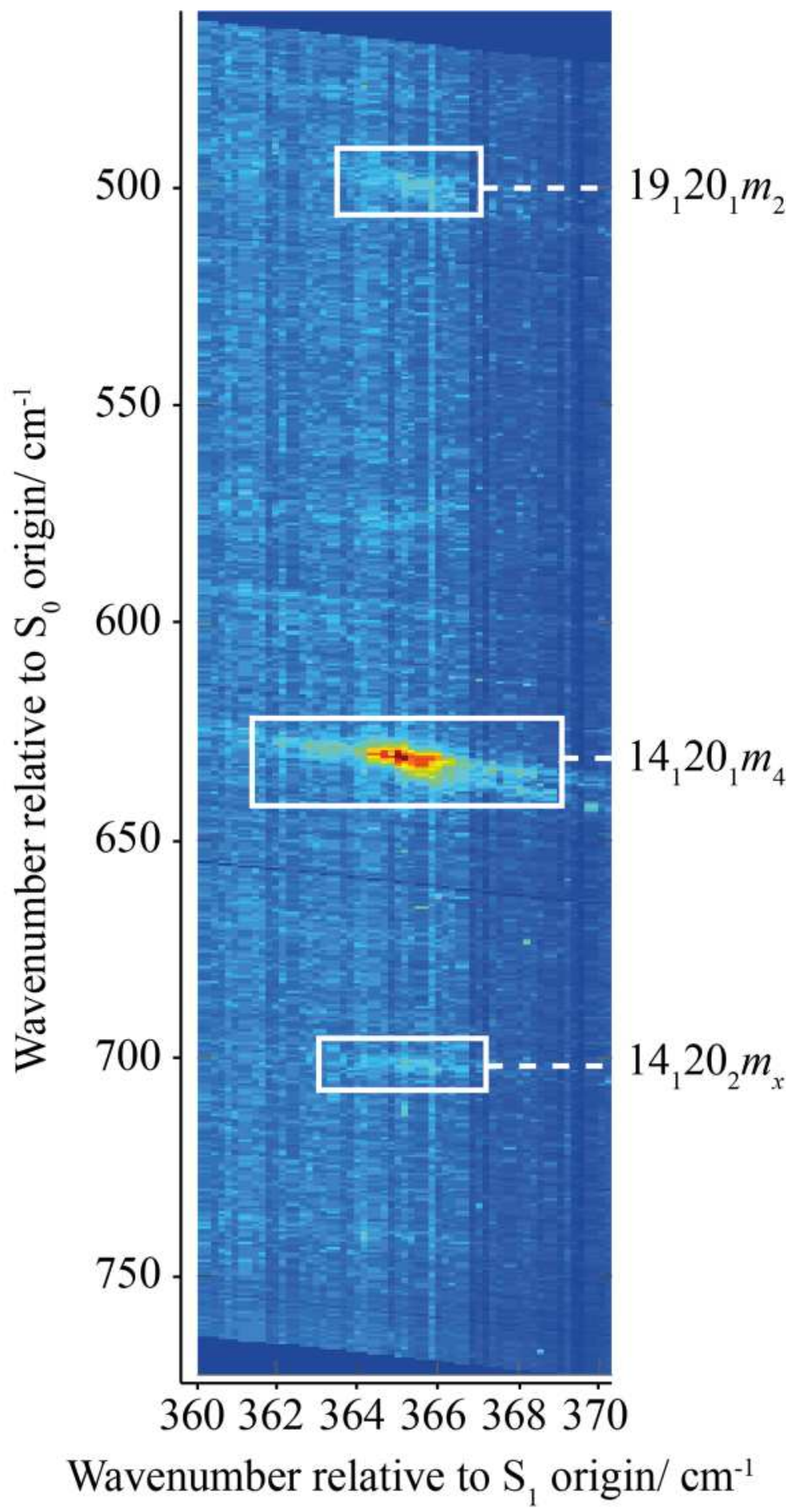


Figure 11

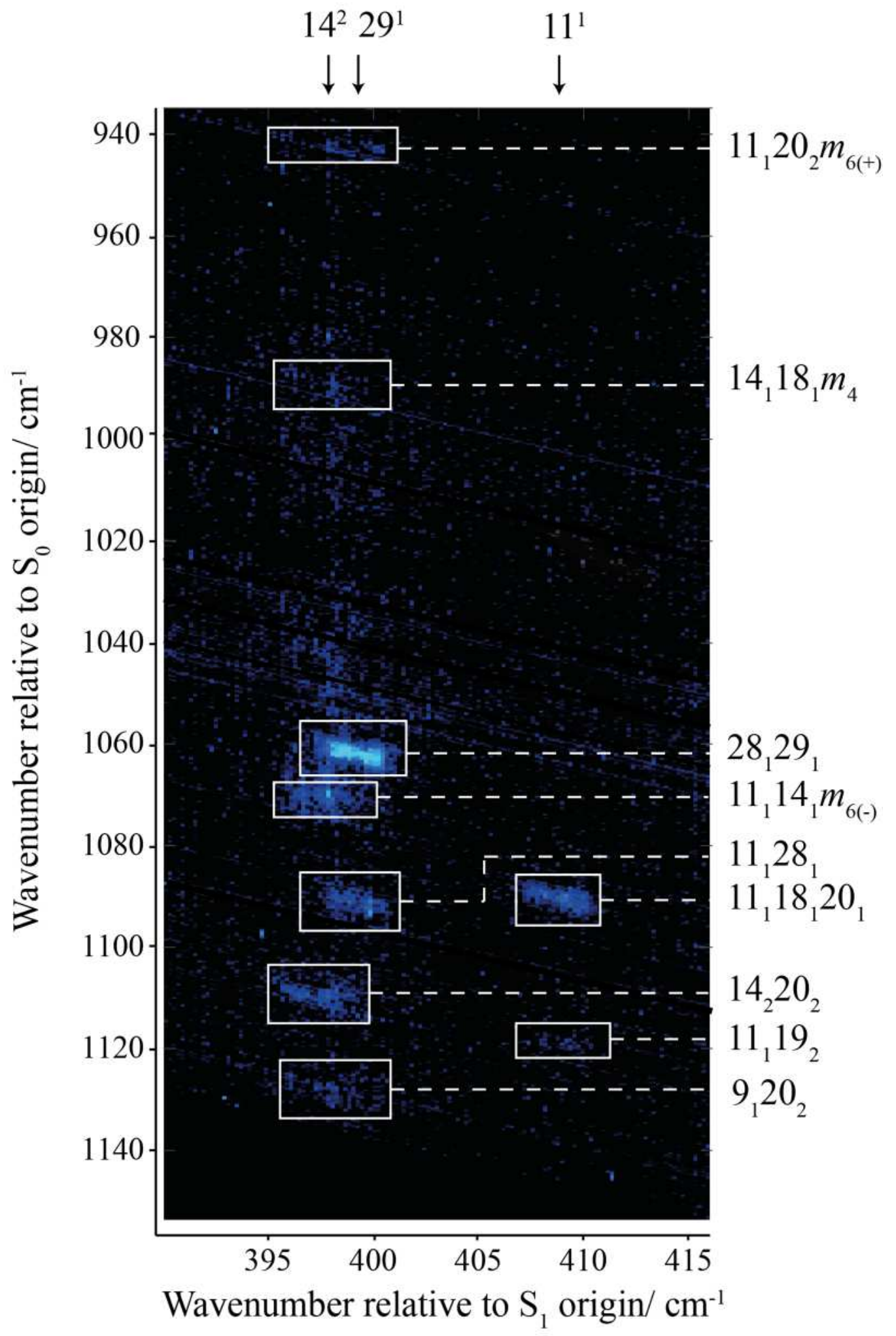


Figure 12

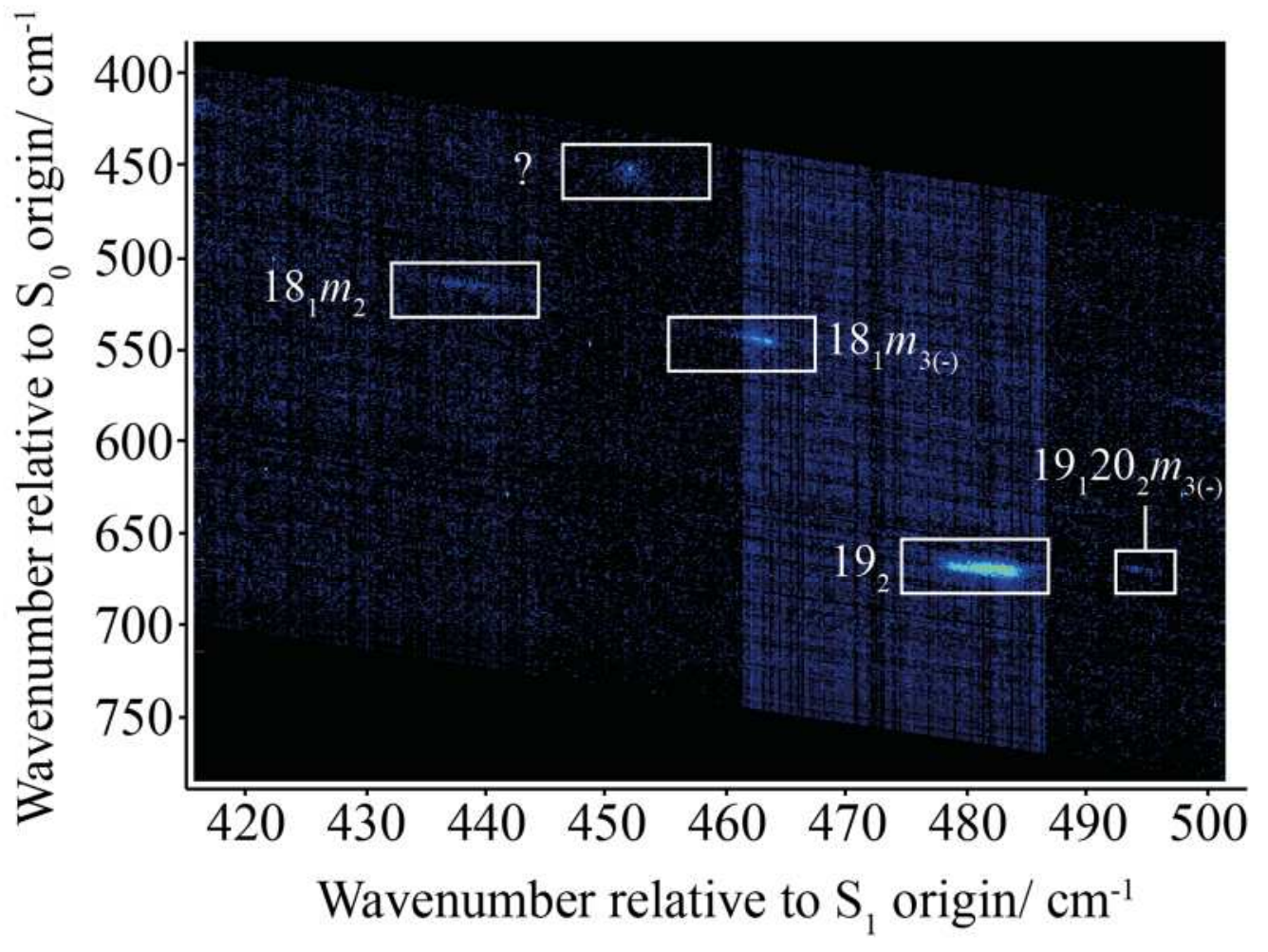




\section{Figure Captions}

Figure 1: The $390-415 \mathrm{~cm}^{-1}$ region of the $S_{1} \leftarrow S_{0}$ excitation spectrum of $p F T$ recorded by (a) REMPI spectroscopy and (b) by vertical integration of the 2D-LIF spectrum across the emission range 350$890 \mathrm{~cm}^{-1}$. The $14^{2}$ and $29^{1}$ bands overlap - see text.

Figure 2: Overview of the main region of the 2D-LIF spectra recorded in the present work. The spectral intensities are represented by colours, with red being the most intense through to blue being the least; black represents the zero background.

Figure 3: Expanded view of the DF spectrum of $p \mathrm{FT}$ in the $0-390 \mathrm{~cm}^{-1}$ region exciting via the origin, and the eigenstates dominated by $29^{1}, 14^{2}$ and $11^{1}$. This region covers the main torsion and vibtor levels associated with the $D_{20}, D_{19}$ and $D_{30}$ vibrations. The appearance of the spectrum via the origin is very similar to that recently published by Gascooke et al. in Ref. 16, where it is discussed in detail.

Figure 4: Expanded view of the DF spectrum of $p F T$ in the $390-1090 \mathrm{~cm}^{-1}$ region exciting via the origin, and the eigenstates dominated by $29^{1}, 14^{2}$ and $11^{1}$. Selected assignments are shown and the spectrum is discussed further in the text together with the 2D-LIF spectra. Note that for the feature marked $14_{1} 20_{2} m_{x} x$ may be 1 and/or 2 - see Section 3.4.3.3. Note that the $14^{2}$ and $29^{1}$ bands overlap and so the respective DF spectra contain small contributions from the overlapped feature.

Figure 5: Expanded view of the DF spectrum of $p \mathrm{FT}$ in the $1090-1390 \mathrm{~cm}^{-1}$ region exciting via the origin, and the eigenstates dominated by $29^{1}, 14^{2}$ and $11^{1}$. Selected assignments are shown and the spectrum is discussed further in the text together with the 2D-LIF spectra. Note that the $14^{2}$ and $29^{1}$ bands overlap and so the respective DF spectra contain small contributions from both levels.

Figure 6: Expanded view of the 2D-LIF spectrum covering the main $\Delta(v, m)=0$ regions, $\left(29^{1}, 29_{1}\right)$ and $\left(11^{1}, 11_{1}\right)$. The approximate positions of the origins of the $14^{2}, 29^{1}$ and $11^{1}$ excitations are indicated at the top of the spectrum. The spectral intensities are represented by colours, with red being the most intense through to blue being the least; black represents the zero background.

Figure 7: Partial LIF spectra obtained by integrating across regions four $\Delta(v, m)=0$ bands: $14^{2}, 29^{1}$, $14^{1} m^{6(-)}$ and $11^{1}$ - see text. Note that the integrations were carried out over a vertical slice that included the main central activity of each band, and owing to different band shapes, and the need to avoid contributions from other features, these slices were not of the same height and so the results would not be accurately representative of relative intensities; thus, we have scaled three of these to the same maximum intensity, and one to half of their intensity for presentational reasons.

Figure 8: (a) Expanded view of the 2D-LIF spectrum covering the main $\left(14^{2}, 14_{2}\right) \Delta(v, m)=0$ regions, and also $\left(11^{1}, 11_{2}\right),\left(29^{1}, 29_{2}\right)$ and $\left(29^{1}, 11_{1} 29_{1}\right)$. The approximate positions of the origins of the $14^{2}$, $29^{1}$ and $11^{1}$ excitations are indicated at the top of the spectrum. (b) Further expanded view showing the two extra features close to the $\left(14^{2}, 14_{2}\right)$ band marked $A$ and $B$ - see text. The spectral intensities are represented by colours, with red being the most intense through to blue being the least; black represents the zero background. 
Figure 9: Expanded view of the 2D-LIF spectrum covering the main $\left(14^{1} m^{6(-)}, 14_{1} m_{6(-)}\right) \Delta(v, m)=0$ regions, and also $\left(29^{1}, 28_{1}\right)$. The approximate positions of the origins of the $14^{2}, 29^{1}$ and $11^{1}$ excitations are indicated at the top of the spectrum. Note that for the feature marked $14_{1} 20_{2} m_{x}, x$ may be 1 and/or 2 - see text. The spectral intensities are represented by colours, with red being the most intense through to blue being the least; black represents the zero background.

Figure 10: Expanded view of the 2D-LIF spectrum covering excitation of the $S_{1} 0^{0}+364 \mathrm{~cm}^{-1}$ band. Note that for the feature marked $14_{1} 20_{2} m_{x}, x$ may be 1 and/or 2 - see text. The spectral intensities are represented by colours, with red being the most intense through to blue being the least; on this scale the zero background appears as a pale blue. Note that the triangular regions at the top right and bottom left of the image are unscanned regions.

Figure 11: Expanded view of the 2D-LIF spectrum covering the higher-wavenumber range 940-1140 $\mathrm{cm}^{-1}$. The approximate positions of the origins of the $14^{2}, 29^{1}$ and $11^{1}$ excitations are indicated at the top of the spectrum. The spectral intensities are represented by colours, with red being the most intense through to blue being the least; black representing the zero background.

Figure 12: Expanded view of the 2D-LIF spectrum covering the excitation wavenumber range $\mathrm{S}_{1} \mathrm{O}^{0}+$ $420-500 \mathrm{~cm}^{-1}$. The spectral intensities are represented by colours, with red being the most intense through to blue being the least; black represents the zero background in most areas, but between $460-485 \mathrm{~cm}^{-1}$ there is a region where the background is different, and appears as pale blue. Note that the triangular regions at the top right and bottom left of the image are unscanned regions. Tentative suggested assignments for the band marked '?' are given in the text. 